\title{
A kinome-wide RNAi screen identifies ALK as a target to sensitize neuroblastoma cells for HDAC8-inhibitor treatment
}

\author{
Jing Shen ${ }^{1}$ - Sara Najafi ${ }^{1,2}$ - Sina Stäble ${ }^{1}$ Johannes Fabian ${ }^{1,3}$ - Emily Koeneke ${ }^{1,2} \cdot$ Fiona R. Kolbinger $^{1,2}$.

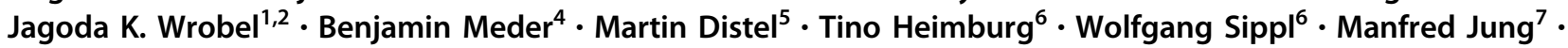 \\ Heike Peterziel $^{1,2} \cdot$ Dominique Kranz $^{8} \cdot$ Michael Boutros $^{8} \cdot$ Frank Westermann $^{9} \cdot$ Olaf Witt $^{1,2,10} \cdot$ Ina Oehme $^{1,2}$
}

Received: 14 September 2017 / Revised: 24 January 2018 / Accepted: 25 January 2018 / Published online: 7 March 2018

(c) The Author(s) 2018. This article is published with open access

\begin{abstract}
The prognosis of advanced stage neuroblastoma patients remains poor and, despite intensive therapy, the 5-year survival rate remains less than $50 \%$. We previously identified histone deacetylase (HDAC) 8 as an indicator of poor clinical outcome and a selective drug target for differentiation therapy in vitro and in vivo. Here, we performed kinome-wide RNAi screening to identify genes that are synthetically lethal with HDAC8 inhibitors. These experiments identified the neuroblastoma predisposition gene $A L K$ as a candidate gene. Accordingly, the combination of the ALK/MET inhibitor crizotinib and selective HDAC8 inhibitors (3-6 $\mu \mathrm{M}$ PCI-34051 or $10 \mu \mathrm{M}$ 20a) efficiently killed neuroblastoma cell lines carrying wildtype $A L K$ (SK-N-BE(2)-C, IMR5/75), amplified $A L K$ (NB-1), and those carrying the activating $A L K$ F1174L mutation (Kelly), and, in cells carrying the activating R1275Q mutation (LAN-5), combination treatment decreased viable cell count. The effective dose of crizotinib in neuroblastoma cell lines ranged from $0.05 \mu \mathrm{M}$ ( $A L K$-amplified) to $0.8 \mu \mathrm{M}$ (wildtype $A L K$ ). The combinatorial inhibition of ALK and HDAC8 also decreased tumor growth in an in vivo zebrafish xenograft model. Bioinformatic analyses revealed that the mRNA expression level of $H D A C 8$ was significantly correlated with that of $A L K$ in two independent patient cohorts, the Academic Medical Center cohort $(n=88)$ and the German Neuroblastoma Trial cohort $(n=649)$, and co-expression of both target genes identified patients with very poor outcome. Mechanistically, HDAC8 and ALK converge at the level of receptor tyrosine kinase (RTK) signaling and their downstream survival pathways, such as ERK signaling. Combination treatment of HDAC8 inhibitor with crizotinib efficiently blocked the activation of growth receptor survival signaling and shifted the cell cycle arrest and differentiation phenotype toward effective cell death of neuroblastoma cell lines, including sensitization of resistant models, but not of normal cells. These findings reveal combined targeting of ALK and HDAC8 as a novel strategy for the treatment of neuroblastoma.
\end{abstract}

Edited by R.A. Knight.

Electronic supplementary material The online version of this article (https://doi.org/10.1038/s41418-018-0080-0) contains supplementary material, which is available to authorized users.

$\square$ Ina Oehme

i.oehme@dkfz.de

1 Clinical Cooperation Unit Pediatric Oncology, German Cancer Research Center (DKFZ) and German Cancer Consortium (DKTK), Heidelberg, Germany

2 Preclinical Program, Hopp Children's Cancer Center at NCT Heidelberg (KiTZ), Heidelberg, Germany

3 Present address: Phenex Pharmaceuticals AG, Heidelberg, Germany

4 Institute for Cardiomyopathies Heidelberg, Heidelberg University, Heidelberg, Germany

5 Innovative Cancer Models, St. Anna Children's Cancer Research Institute, Vienna, Austria
6 Institute of Pharmacy, Martin-Luther University of HalleWittenberg, 06120 Halle/Saale, Germany

7 Institute of Pharmaceutical Sciences, University of Freiburg, 79104 Freiburg, Germany

8 Division of Signaling and Functional Genomics, German Cancer Research Center and Heidelberg University, Department for Cell and Molecular Biology, Medical Faculty Mannheim, Heidelberg, Germany

9 Research Group Neuroblastoma Genomics, German Cancer Research Center, Heidelberg, Germany

10 Department of Pediatric Oncology, Hematology and Immunology, University of Heidelberg Medical Center, Heidelberg, Germany 


\section{Introduction}

Neuroblastoma is the most common extracranial solid tumor in children and is derived from precursor cells of the peripheral sympathetic nervous system. The 5-year overall survival probability of high-risk neuroblastoma patients is less than 50\% [1]. Moreover, chemotherapy-treated patients struggle with therapy-related immediate and long-term toxicities (reviewed in Brodeur [2]). Thus, more neuroblastoma-specific therapeutic approaches focusing on oncogenic molecular targets are required to improve therapeutic efficacy, reduce toxicity and avoid long-term side effects.

Small molecules that influence gene transcription are also of high interest for the treatment of cancer. One class of drugs in this category are histone deacetylase (HDAC) inhibitors, such as vorinostat (SAHA: suberoylanilide hydroxamic acid), the first clinical HDAC inhibitor approved by the FDA for the treatment of refractory cutaneous T-cell lymphoma [3]. Most inhibitors of HDAC enzymatic activity bind to the highly conserved catalytic domain and hence unselectively inhibit the activity of all zinc-dependent HDAC family members [4]. The enzyme family is grouped into four classes based on their homology to yeast HDACs. In the strict sense, HDACs are more general lysine deacetylase (KDACs), as these enzymes remove acetyl groups from lysine residues of numerous nuclear and cytosolic proteins, affecting gene transcription as well as many cellular pathways [5, 6]. Three of the four classes (class I, II, and IV) have a zinc-dependent catalytic mechanism and constitute the so-called classical HDACs. HDAC family member 8 (HDAC8) together with HDACs 1, 2, and 3 comprise class I [7]. HDAC8 may be an attractive selective target with specific features, as crystal structure analysis revealed a unique second metal binding site in close proximity to the main catalytic domain [8], allowing the design of HDAC8selective inhibitors. Furthermore, we have previously shown that HDAC8 expression is correlated with advanced tumor stage and poor outcome in neuroblastoma patients [9]. Selective inhibition of HDAC8 slows neuroblastoma growth, induces a more differentiated phenotype and serves as a potent enhancer of retinoic acidmediated anti-neuroblastoma activity both in vitro and in vivo [10].

RNA interference (RNAi) screens are commonly applied to identify novel limiting factors for drug responsiveness and to unravel targeted combinations of specific drugs to overcome these limitations [11]. Here, we used a kinomewide RNAi screen to identify new combinations that enhance the sensitivity of neuroblastoma to HDAC8 inhibitors. We identified receptor tyrosine kinases (RTKs), such as anaplastic lymphoma kinase (ALK), as druggable neuroblastoma cell survival activators that can be targeted by treatment with small molecule inhibitors, thus sensitizing neuroblastomas to HDAC8 inhibition.

\section{Results}

\section{Kinome-wide siRNA screen identifies druggable kinases for the sensitization of neuroblastoma cells to HDAC8 inhibitor treatment}

Although HDAC8 inhibitor application significantly slows tumor growth in vivo, the therapeutic effect of treatment with a single HDAC8 inhibitor is not sufficient to induce complete tumor regression, as desired [10]. Cell culture experiments revealed a very strong response in sensitive neuroblastoma cell line IMR-32, whereas other neuroblastoma cell lines investigated responded to HDAC8 inhibition with cell cycle arrest and signs of differentiation rather than cell death (Fig. 1a and [10]). To detect HDAC8 co-dependencies and druggable co-targets, we performed an HDAC8 inhibitor synthetic lethal screen using the siKINOME SMARTpool library (Dharmacon) using short interfering RNA (siRNA) pools consisting of four single siRNAs each, targeting approximately 780 human protein kinases and kinase-associated genes. The SK-N-BE(2)-C cell line was chosen for screening because it exhibits an intermediate response to HDAC8 inhibitor treatment and is one of the most aggressive neuroblastoma cell lines derived from a relapsed $M Y C N$-amplified tumor with a TP53 mutation [12]. Three treatment conditions were applied: solvent and treatment with two structurally different selective HDAC8 inhibitors (Cpd2 [13] and PCI-34051 [14]). Comparison of duplicate experiments revealed high reproducibility of the screen (Supplementary Figure 1A). The screen was optimized to detect sensitizing ("lethal") and inhibitory ("rescue") effects by incubating cells with the IC50 concentration of HDAC8 inhibitors $(40 \mu \mathrm{M} \mathrm{Cpd} 2$ and $4 \mu \mathrm{M}$ PCI-34051). After $96 \mathrm{~h}$, neuroblastoma cell viability was assessed by Cell Titer Glo (CTG) assays (Fig. 1b). Data were normalized to the respective treatment with dimethyl sulfoxide (DMSO) or HDAC8i (Supplementary Figure 1B-D). A hit was defined as HDAC8i-normalized treatment minus DMSO-normalized treatment $>60,000$ RLU (=rescue hit; orange shading) or $<-60,000$ RLU (=lethality hit; green shading) (Fig. 1c; Supplementary Table 1). This cut-off separates the candidates of interest (blue HDAC8i \#1, red HDAC8i \#2) from the expected treatment effect (black circles of all treatments). Finally, hits were defined as only those candidates whose effects were reproducible for both replicates and both HDAC8 inhibitors. In total, the screen identified 84 common hits (Fig. 1d): 41 rescue hits, and 43 lethality hits (Supplementary 
Fig. 1 Kinome-wide RNAi screen for the identification of HDAC8 inhibitor codependencies. a IMR-32, SK-N$\mathrm{BE}(2)-\mathrm{C}, \mathrm{LAN}-5$, Kelly and NB1 cell lines were treated with increasing concentrations of Cpd 2 and then monitored for 96 $\mathrm{h}$ after treatment for cell death using trypan blue staining (dead cells: trypan blue-positive cells). b Schematic of the RNAi screen. c Hit plot displaying "rescue hits" and "lethality hits" above or below the expected treatment effect area. d Venn diagram showing the overlay of identified hits between both HDAC8 inhibitors. e Horizontal bar diagram displaying the $P$-values for significantly enriched pathways (REACTOME; http://www.reactome.org/). f, $\mathbf{g}$ "Rescue hit" examples from the RNAi screen. The bar diagrams display CTG activity upon $96 \mathrm{~h}$ of HDAC8i \#1 (f) and \#2 (g) treatment normalized for each siRNA pool. b-d, f, g HDAC8i: HDAC8 inhibitor; HDAC8i \#1: Cpd2 (red), HDAC8i \#2: PCI34051 (blue)
A

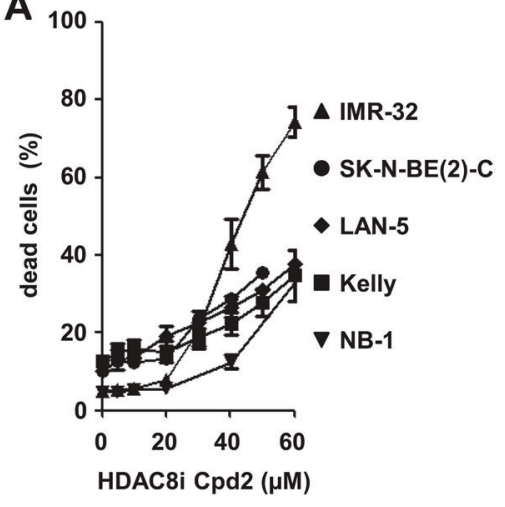

B
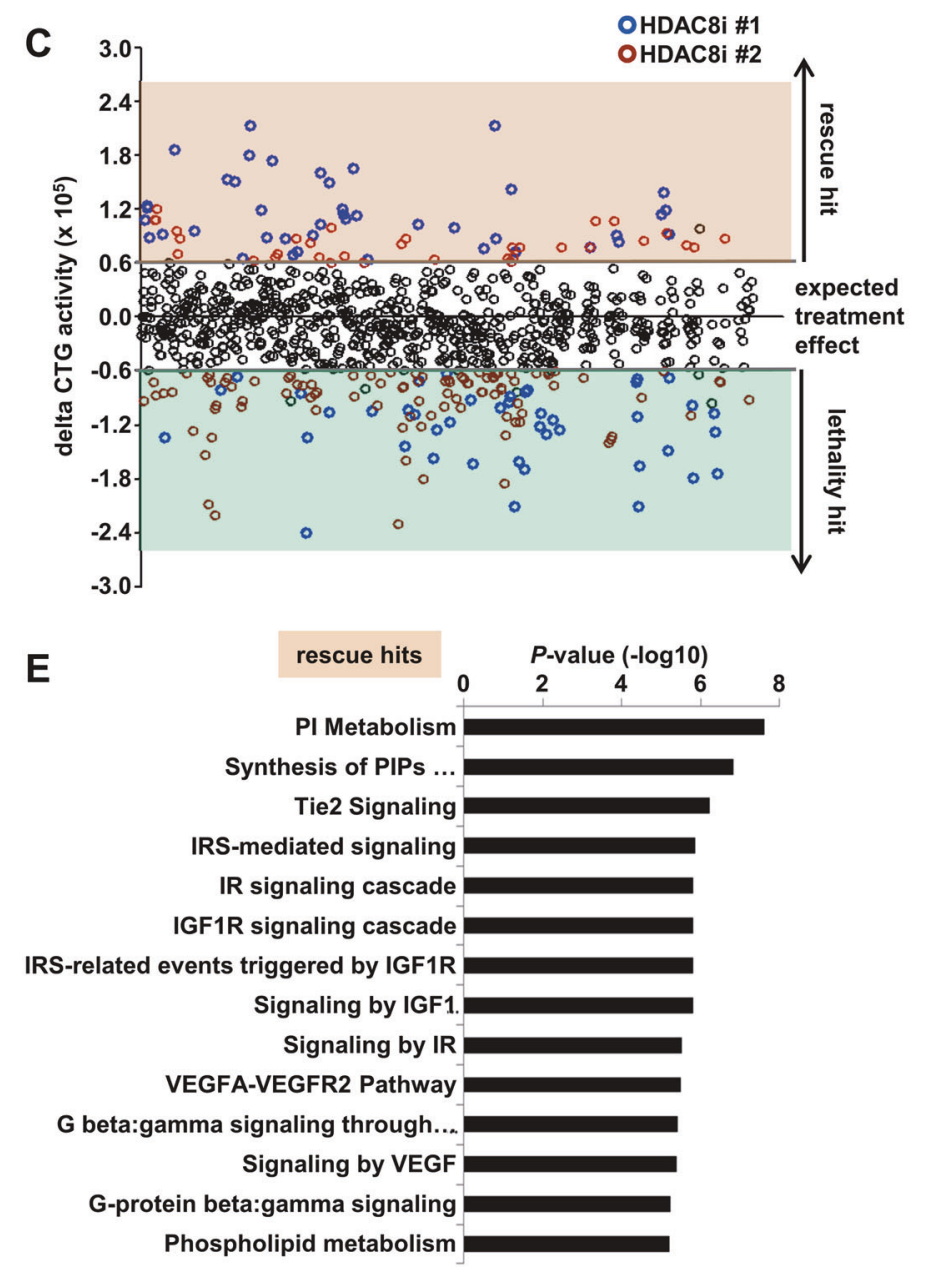

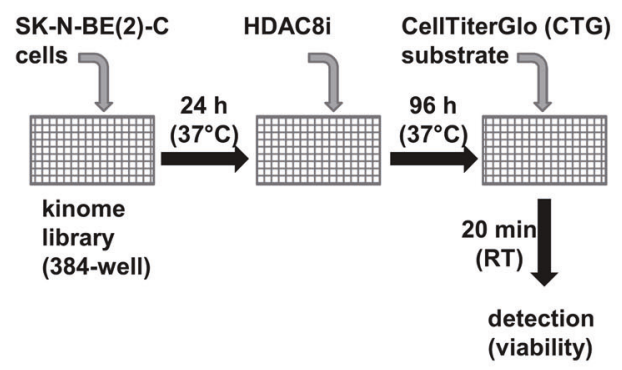

D HDAC8i

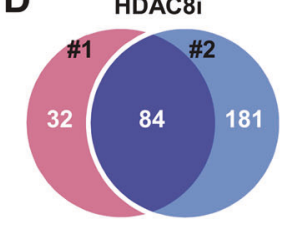

$\mathbf{F}$

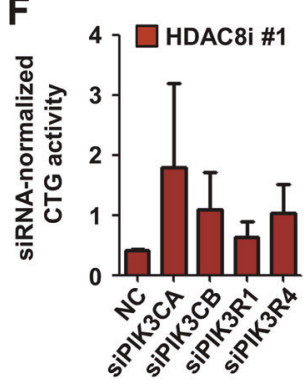

G

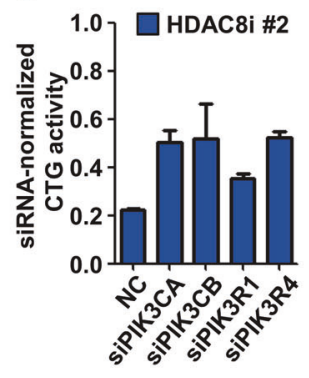

Table 1). Analysis of the "rescue hit list" by gene ontology enrichment analysis with GOrilla revealed the overrepresentation of phosphatidylinositol kinase and phosphatidylinositol bisphosphate kinase activity (Supplementary Figure 1E), which was confirmed by pathway analysis with REACTOME (cut-off $p<0.00001$; Fig. 1e). This suggests that PI3K pathway inactivation abolishes the antineuroblastoma effect of the HDAC8 inhibitor, exemplarily shown for knockdown of PIK3CA, PIK3CB, PIK3R1 and PIK3R4 in Fig. 1f, g. REACTOME pathway analysis of the "lethality hit list" revealed axon guidance (e.g., RET and Ephrin signaling), CREB-PKC/MAPK signaling and NGF signaling to be significantly enriched (Fig. 2a). A protein class analysis (PANTHER) [15] indicated an enrichment of non-receptor serine-threonine kinases and RTKs among the lethality hits (Fig. 2b). Figure 2c shows the treatment sensitizing effect of knockdown of the eight RTKs (ERBB2, FLT1, ALK, EPHA2, EPHB2, EPHB4, KIT, and TYRO3). Besides HDAC8 inhibitor-specific hits, general toxicity hits of neuroblastoma, such as the known effectors of cell 
Fig. 2 Identification of HDAC8 inhibitor sensitization hits. a Horizontal bar diagram displaying significantly enriched signaling pathways for "lethality" hits detected via REACTOME pathway analysis. b Horizontal bar diagram displaying the distribution of protein classes for "lethality" hits based on the PANTHER classification system (pthr 24416). Only classes with at least two kinases are shown. PK, protein kinase. RTK, receptor tyrosine kinase. GEF, guanylnucleotide exchange factor. c "Lethality hit" examples from the RNAi screen. The bar diagram displays CTG activity upon knockdown of the eight RTKs, identified in (b), $96 \mathrm{~h}$ of HDAC8i treatment. Data are normalized for treatment with each compound
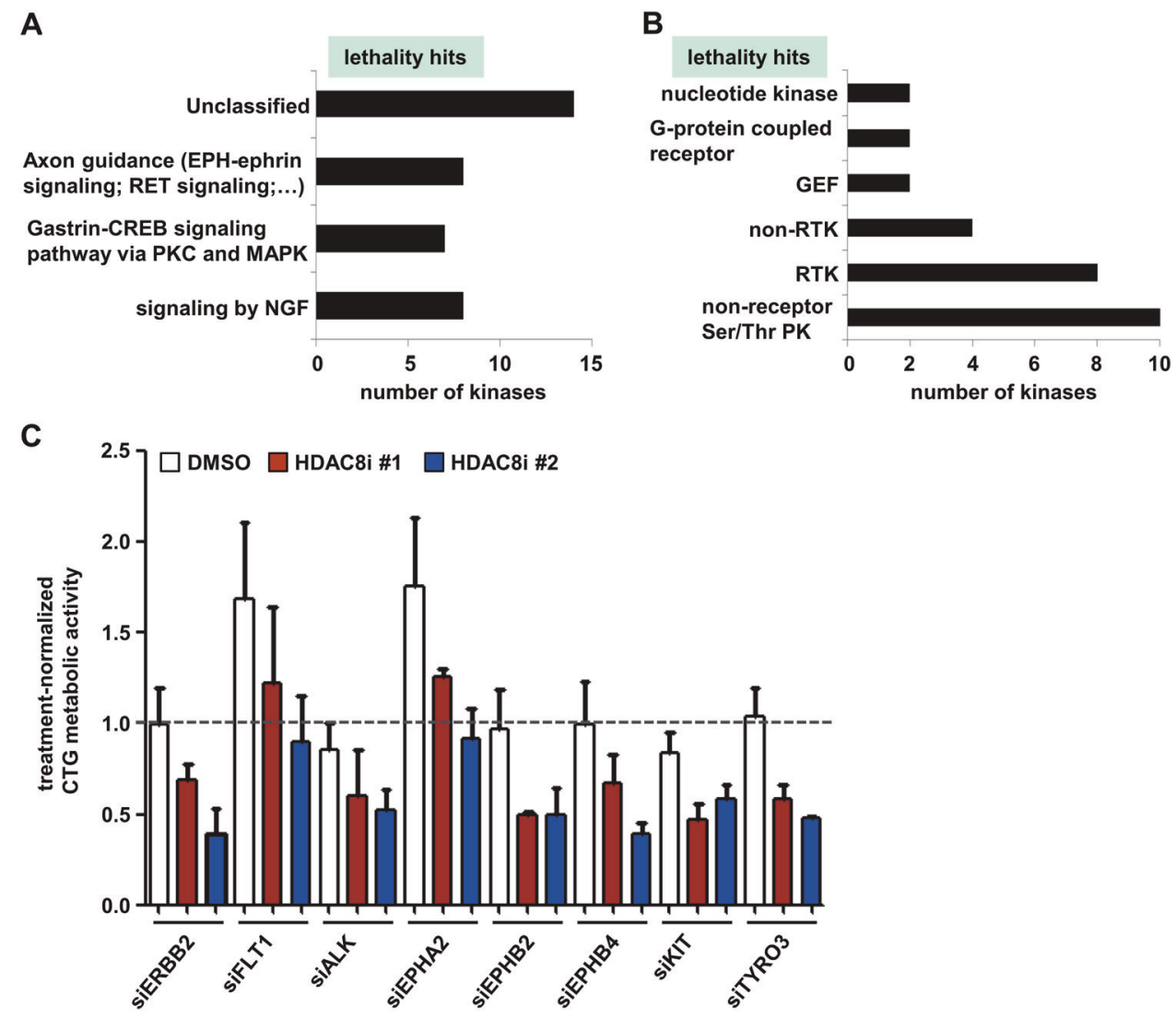

viability, PLK1 [16] and WEE1 [17, 18], were identified in the screen and are listed in Supplementary Table 2. Overall, the kinome-wide RNAi screen identified the PI3K pathway to be involved in HDAC8 inhibitor-mediated anti-neuroblastoma effects and identified additional druggable RTKs, such as ERBB2 and ALK, as targets to sensitize neuroblastoma to HDAC8 inhibitor treatment.

\section{Combined ALK and HDAC8 inhibitor treatment on ALK wild-type neuroblastoma cells}

We selected one RTK, ALK, for subsequent studies, as the gene encoding for ALK was identified as a major familial neuroblastoma predisposition gene [19] and can be targeted by US Food and Drug Administration (FDA)-approved drugs. The ALK/MET/ROS1 inhibitor crizotinib [20] is approved for the treatment of non-small cell lung carcinoma (NSCLC) with ALK translocations [21-23] and is also being tested in neuroblastoma (clinical trial: NCT00939770) [24]. Furthermore, the ALK inhibitor LDK378 is being investigated in clinical trials for the treatment of pediatric malignancies with a genetic alteration of ALK (NCT01742286). Schulte et al. [25] recently demonstrated high ALK expression in primary neuroblastoma as a determining factor of an unfavorable phenotype.
ALK expression studies using two publicly available neuroblastoma cohorts in the R2 database [26], the Academic Medical Center (AMC) cohort with 88 patient samples and the large cohort of neuroblastoma cases $(n=649)$ from the German Neuroblastoma Trial [27], revealed a strong correlation with HDAC8 expression (Fig. 3a, b). When the large cohort was separated by stage (Fig. 3c-f), a strong, significant correlation was only found in International Neuroblastoma Staging System (INSS) stage 4 patients (Fig. 3e). Accordingly, the co-expression of both genes, $A L K$ and $H D A C 8$, was correlated with poor survival of neuroblastoma patients in both cohorts, with long-term overall and event-free survival below 50\% (Table 1; Fig. $3 g, h)$. Cox regression analysis identified that, even after accounting for the effects of stage, high HDAC8 coexpressed with ALK is a significant risk factor for poor outcomes in neuroblastoma patients (Supplementary Table 3), further supporting the investigation of combining an HDAC8 inhibitor with an ALK inhibitor for neuroblastoma treatment.

To further validate ALK as a suitable target for the sensitization of neuroblastoma cells to HDAC8 inhibitor treatment, SK-N-BE(2)-C cells were transfected with the two most effective single ALK siRNAs and treated with the HDAC8 inhibitor PCI-34051 [14] (Fig. 4a), and vice versa, SK-N-BE(2)-C cells transfected with an siRNA pool against 
A

total cohort $(n=88)$

alive $(n=55) \quad$ dead $(n=33)$

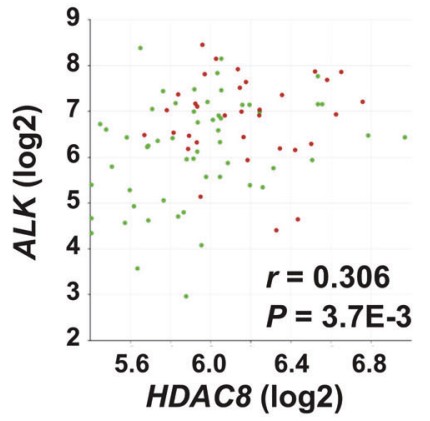

D INSS stage $3(n=91)$

alive $(n=72) \quad$ dead $(n=19)$

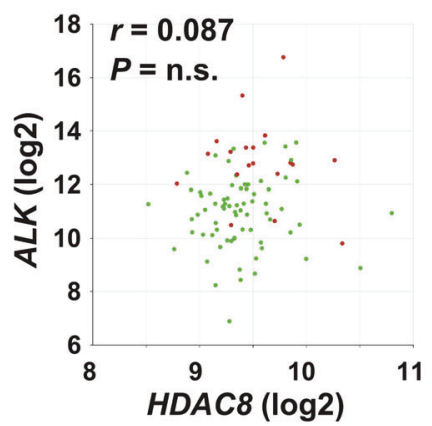

G

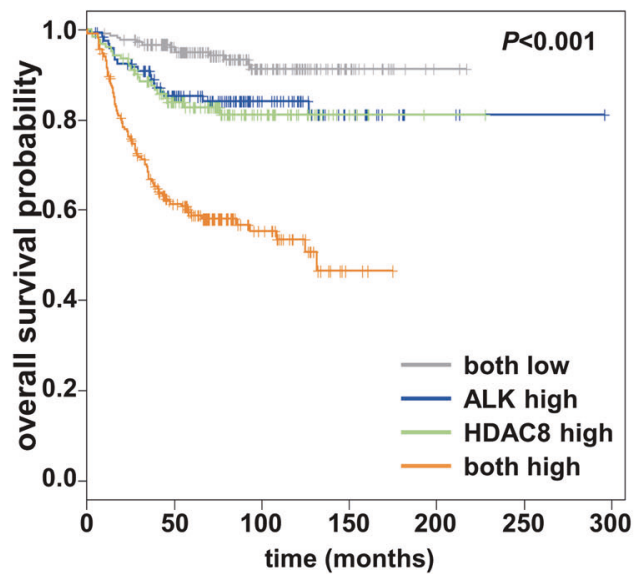

Fig. 3 Correlated ALK and HDAC8 expression in INSS stage 4 patients. (a-f) Scatter plots are shown for the expression of HDAC8 and ALK in neuroblastoma patient cohorts; green dots, alive; red dots, dead. The R2 platform was used for the calculation, and the AMC cohort "Neuroblastoma public-Versteeg -88" (MAS5.0-u133p2) (a) as well as the Cologne cohort "Neuroblastoma-Kocak -649" (custom.ag44kcwolf) (b-h) was the source of the data. The following probe sets were used: ALK 208211_s_at and HDAC8 223345_at (AMC) and ALK UKv4_A_23_P324304 and HDAC8 UKv4_A_P254965 (Kocak). INSS: International Neuroblastoma Staging System. a Whole cohort, Versteeg. b Whole cohort, Kocak. c INSS stage 1,2 . d iNSS stage 3 . e INSS stage 4 . f INSS stage $4 \mathrm{~s}$. g Kaplan-Meier curves are shown for the overall survival of
C

INSS stage 1, 2 ( $n=266)$

alive $(n=259) \bullet \operatorname{dead}(n=7)$

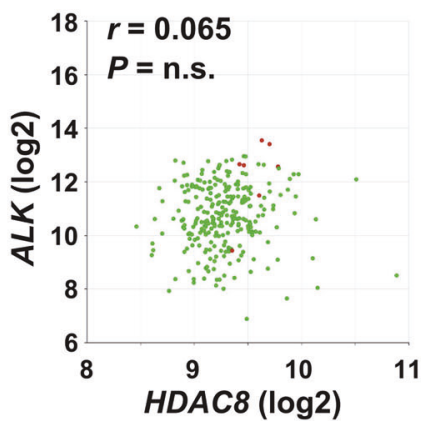

$\mathbf{F}$

INSS stage $4 s(n=78)$ alive $(n=72) \bullet$ dead $(n=6)$

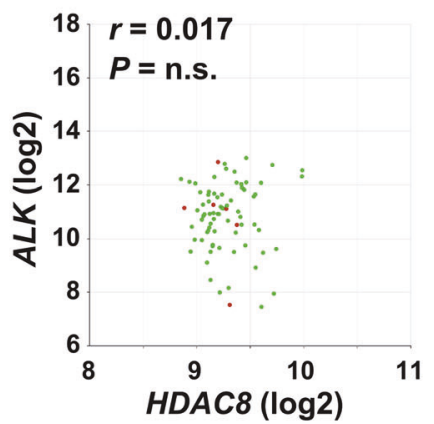

$\mathrm{H}$

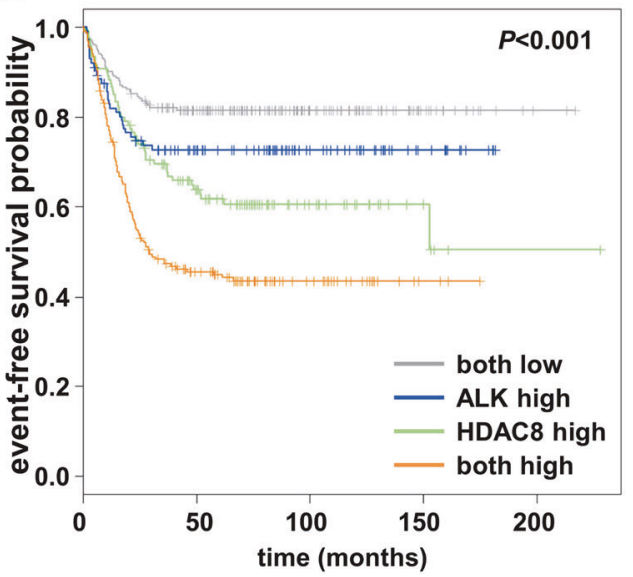

neuroblastoma patients with tumors expressing low levels of both HDAC8 and ALK (both low, gray, $n=205$ ), low levels of HDAC8 and high levels of ALK (ALK high, blue, $n=121$ ), high levels of HDAC8 and low levels of ALK (HDAC8 high, green, $n=122$ ), or high levels of both HDAC8 and ALK (both high, orange, $n=201$ ). (h) Kaplan-Meier curves are shown for the event-free survival of neuroblastoma patients with tumors expressing low levels of both HDAC8 and ALK (both low, gray, $n=201$ ), low levels of HDAC8 and high levels of ALK (ALK high, blue, $n=112$ ), high levels of HDAC8 and low levels of ALK (HDAC8 high, green, $n=119$ ), or high levels of both HDAC8 and ALK (both high, orange, $n=196$ ). $\mathbf{g}$, h The $p$-value was determined using a log-rank test. Median expression was used as the cut-off 
Table 1 Ten year Kaplan-Meier survival probability based on HDAC8 and ALK expression

\begin{tabular}{llll}
\hline & & Overall survival (95\% CI) & Event-free survival (95\% CI) \\
\hline HDAC8 low/ALK low & German NB Trial & $0.911(0.866-0.959)$ & $0.815(0.763-0.871)$ \\
& AMC Cohort & $0.821(0.691-0.976)$ & $0.75(0.606-0.929)$ \\
HDAC8 high/ALK high & German NB Trial & $0.534(0.458-0.623)$ & $0.434(0.369-0.512)$ \\
& AMC Cohort & $0.481(0.326-0.712)$ & $0.418(0.263-0.663)$ \\
\hline
\end{tabular}

CI confidence interval, German NB Trial German Neuroblastoma Trial; AMC Academic Medical Center (University of Amsterdam)

Survival rates calculated using Kaplan-Meier estimator. "Low" expression indicates an expression level below the median expression for that gene. "High" expression reflects expression above the median

HDAC8 (Fig. 4b) were treated with crizotinib. In both conditions, combination treatment significantly reduced cellular viability, which served as an indicator of antitumoral effects. Regarding the reduction in cell viability, the IC50 of crizotinib was determined to be $0.86 \mu \mathrm{M}$ in SK-N$\mathrm{BE}(2)-\mathrm{C}$ cells (Fig. 4c). The peak plasma concentration of crizotinib has been estimated to be approximately $1.4 \mu \mathrm{M}$ in patients [24]. The combination of $0.8 \mu \mathrm{M}$ crizotinib with 6 $\mu \mathrm{M}$ PCI-34051 increased the amount of dead SK-N-BE(2)C cells from approximately 20 up to $40 \%$ (Fig. 4c). We next performed colony formation assays to further evaluate the potential therapeutic effects of the combined treatment against neuroblastoma cells. Significantly fewer colonies were formed in the combination treatment groups compared to the single treatment group (Fig. $4 \mathrm{~d}$, e). In line with this result, the combination of an HDAC8 inhibitor and crizotinib impaired tumor growth in the SK-N-BE(2)-C zebrafish xenograft in vivo model (Fig. 4f, Supplementary Figure 2A). Engraftment of human tumor cells into zebrafish, which lack an adaptive immune system in the first month of life, is an effective method for early preclinical drug screening [28]. The transparency of zebrafish embryos allows tracking of fluorescently-labeled SK-N-BE(2)-C cells and monitoring of tumor formation and progression using confocal microscopy. The efficacy of HDAC8 inhibition, crizotinib and the combination was assessed by evaluating tumor volume change, quantified via semiautomated image analysis, from day one (start of treatment) to day three (end of experiment) post-implantation. Here, we used the novel compound 20a, a hydroxamate-based inhibitor selective for HDAC8, not targeting HDAC1-3 [29], since PCI-34051 was toxic to zebrafish embryos (Supplementary Figure 2B). Overall, our results imply sensitizing effects of the combinatorial treatment of crizotinib with HDAC8 inhibition in SK-N-BE(2)-C cells.

\section{Dual targeting of ALK and HDAC8 also eliminates neuroblastoma cells harboring ALK-activating genetic aberrations}

To expand the panel of investigated cell lines, we determined the ALK expression status of five neuroblastoma cell lines, a cell line from a non-neuroblastoma pediatric embryonal tumor (RD, rhabdomyosarcoma) [30], and a non-malignant but proliferative fibroblast line from an infant donor (VH7) [10]. All neuroblastoma cell lines expressed ALK, with strong expression in LAN-5 cells and amplification in NB-1 cells, whereas the other target of crizotinib, c-MET, was not expressed. However, both nonneuroblastoma control lines (RD and VH7) expressed c-MET while ALK expression was not detectable (Fig. 5a). ALK phosphorylation was detected in Kelly, LAN-5, and NB-1 cells and to a much weaker extent in SK-N-BE(2)-C cells (Fig. 5a). As Kelly cells harbor the constitutively active F1174L ALK mutation, which confers primary resistance to the ALK inhibitor crizotinib [31], we tested our treatment combination in these cells and in NB-1 cells harboring $A L K$ and $M Y C N$ amplification [32]. In colony formation assays, combined treatment of cells with PCI34051 and crizotinib significantly impaired the ability of both cell lines to form colonies (Fig. 5b, c). The combined treatment of Kelly cells with PCI-34051 and crizotinib enhanced cell death to approximately $35 \%$ (Fig. 5d). Significantly higher caspase-3 (DEVDase) activity was observed in the combination treatment group compared to the single treatments in Kelly (ALK F1174L) and NB-1 (ALK-amplified) cells (Supplementary Figure 3A), and the proportion of cells in the subG1 area of the cell cycle was significantly enriched in the combination treatment group (Supplementary Figure 3B). The application of a pancaspase inhibitor (zVAD.fmk) significantly reduced the amount of dead cells in the combination-treated group (Supplementary Figure 3C), demonstrating that the combination treatment triggers caspase-mediated programmed cell death.

To ensure target specificity, we exchanged crizotinib for LDK378, and PCI-34051 for the novel HDAC8-selective compound 20a [29], and similar results were obtained (Supplementary Figure 3D-F). Since the R1275Q ALK mutation occurs frequently in neuroblastoma, we also tested combinatorial inhibition in LAN-5 cells. Significantly fewer viable cells remained after treatment with the combination, however, treatment with crizotinib alone was already quite effective in these cells (Supplementary Figure 3G). 
To address the role of the neuroblastoma-specific oncogene $M Y C N$, which cooperates with activated ALK during neuroblastoma pathogenesis [33, 34], the inducible IMR5/ 75 (ALK-amplified) shMYCN knockdown system was utilized [35]. Cells in both conditions, shMYCN off (high MYCN) and shMYCN on (low MYCN), were treated with the HDAC8 inhibitor, crizotinib or the combination of both. The combinatorial treatment with both inhibitors resulted in increased cell death in IMR5/75 cells. This sensitization effect was weaker with the knockdown of MYCN (Fig. 5e), suggesting a sensitizing function of $M Y C N$ amplification for the dual targeting approach. Of note, though the average
A
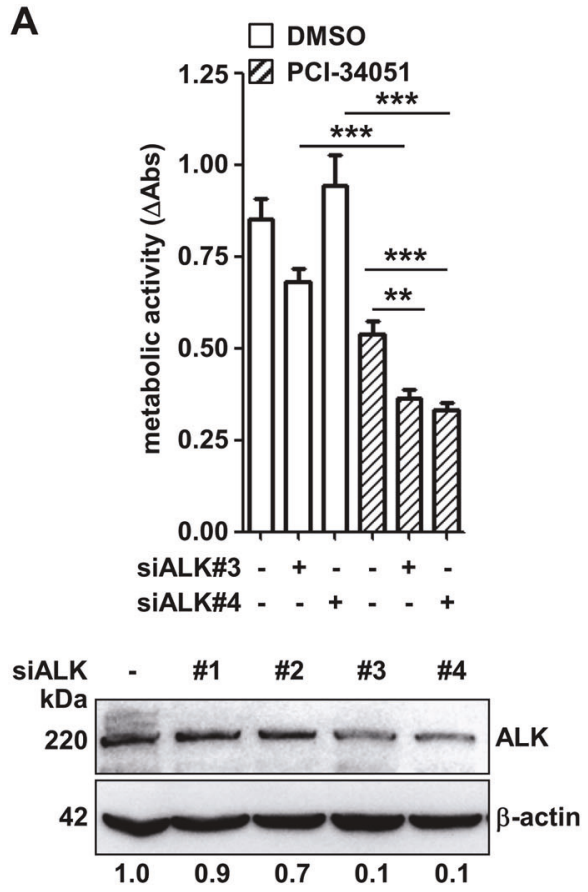

D

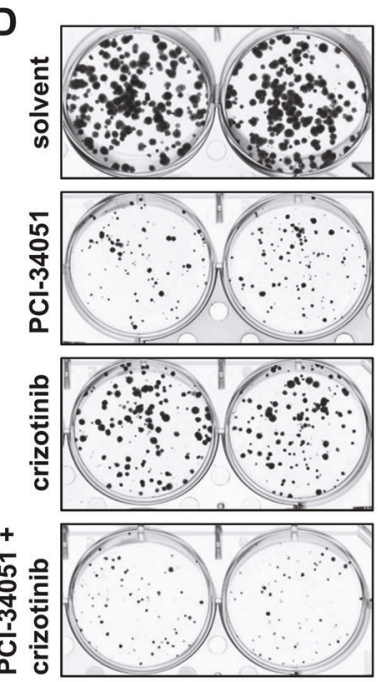

E
B
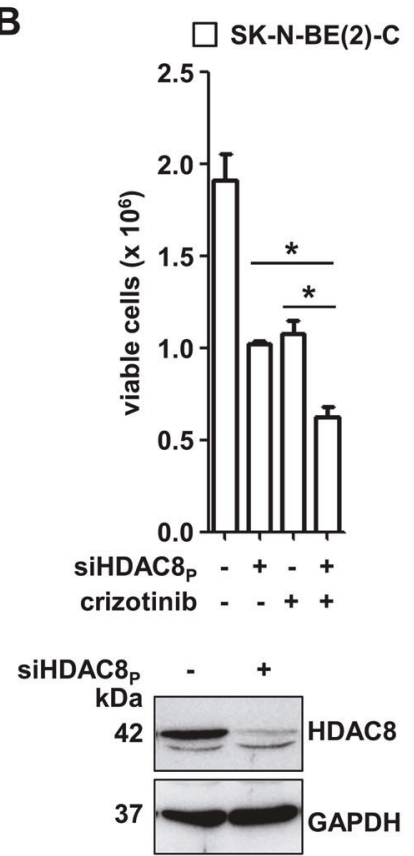

C
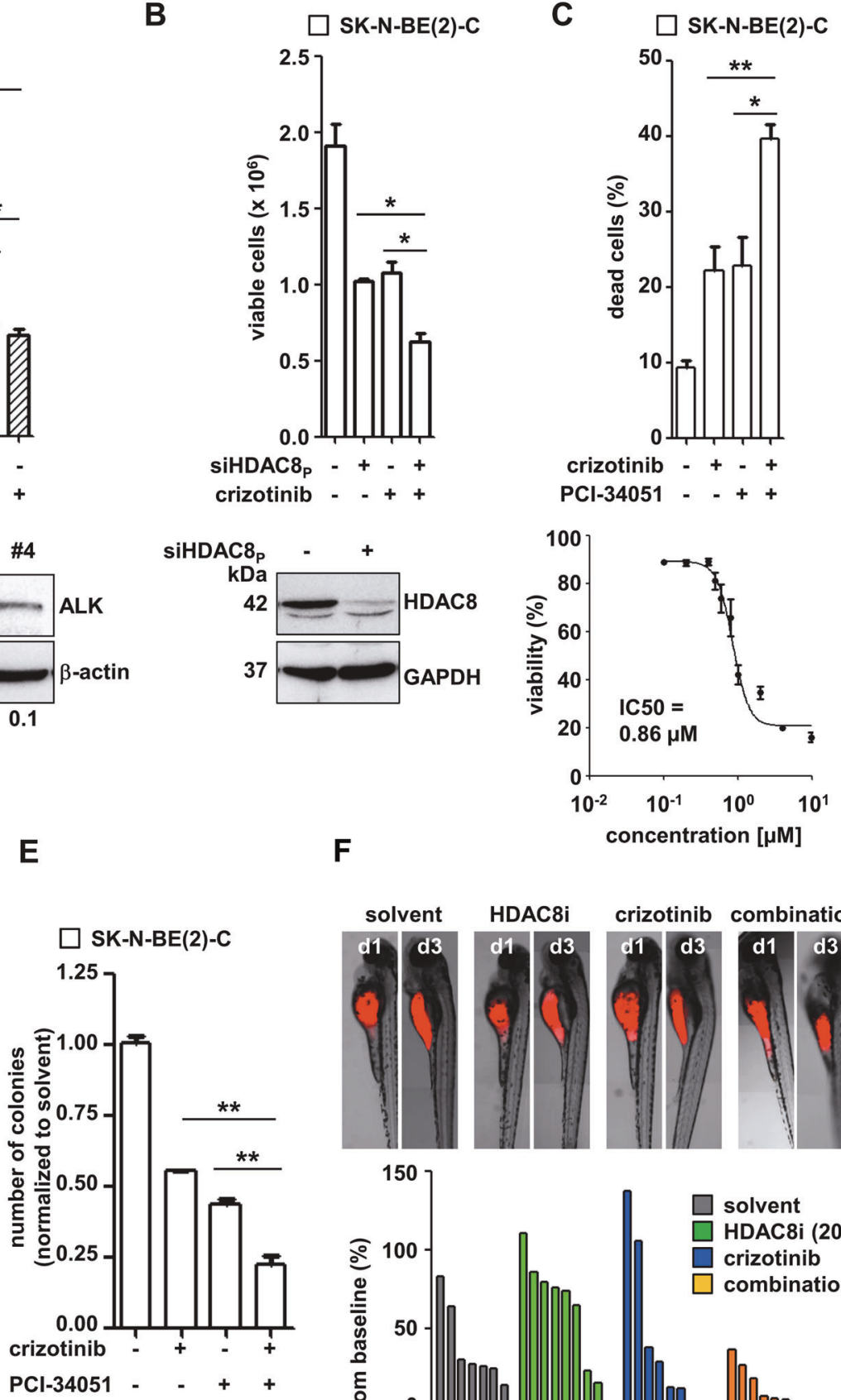

F

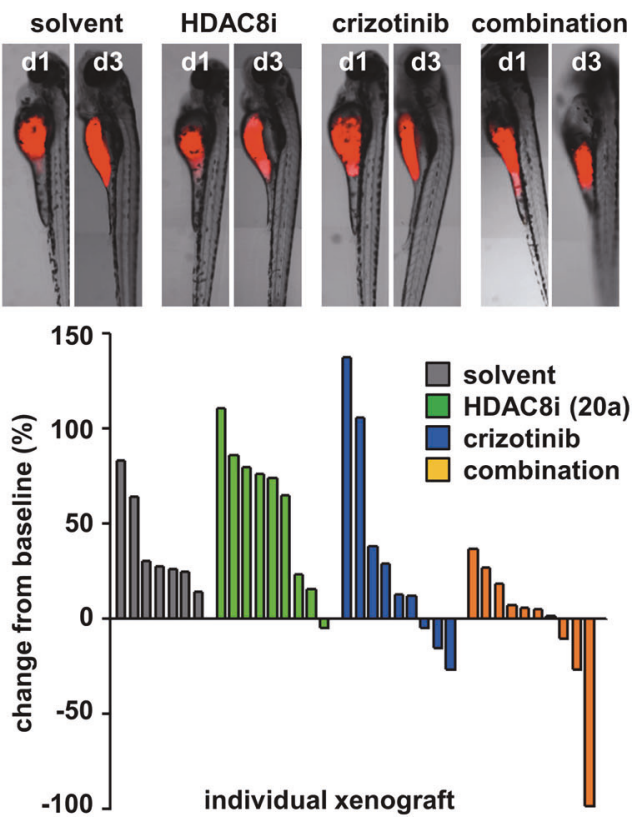


Fig. 4 Validation of ALK as a target for HDAC8 inhibitor sensitization. a Upper: The SK-N-BE(2)-C cell line was transfected with ALK siRNA \#3 and \#4 or negative control siRNA (NC) and $48 \mathrm{~h}$ after transfection treated with $6 \mu \mathrm{M}$ PCI-34051 for $96 \mathrm{~h}$. Metabolic activity was measured by an WST-8 assay and is shown relative to untransfected, untreated cells. Lower: Western blot displaying ALK levels 72 $\mathrm{h}$ after transfection with siRNAs \#1-4. Actin served as a loading control. b Upper: The SK-N-BE(2)-C cell line was transfected with HDAC8 siRNA pool (siHDAC8 8 : siRNA \#1 and \#2) or negative control siRNA (minus sign) and $24 \mathrm{~h}$ later was treated with crizotinib $(0.8 \mu \mathrm{M}, 96 \mathrm{~h})$. The number of viable cells was assessed by automated cell counting and trypan blue exclusion. Lower panel: Western blot displaying HDAC8 levels $72 \mathrm{~h}$ after transfection. GAPDH served as a loading control. c Upper panel: SK-N-BE(2)-C cells were treated with crizotinib $(0.8 \mu \mathrm{M})$ alone or in combination with PCI-34051 $(6 \mu \mathrm{M})$ and then monitored for $96 \mathrm{~h}$ after treatment for cell death using trypan blue staining (dead cells: trypan blue-positive cells). Lower: SK-N-BE (2)-C cells were treated $24 \mathrm{~h}$ post-seeding with various concentrations of crizotinib for $96 \mathrm{~h}$. IC50 values were calculated using GraphPad Prism. Viability (\%) was assessed by automated cell counting and trypan blue exclusion. d SK-N-BE(2)-C cells (800 cells/well) were treated with crizotinib $(0.8 \mu \mathrm{M})$ alone or in combination with PCI$34051(6 \mu \mathrm{M})$, and colonies were stained after 10 days. e Bar diagram displaying the quantified colonies. $\mathbf{f}$ Upper: representative pictures of zebrafish larvae with tumors formed by fluorescently-labeled SK-N$\mathrm{BE}(2)-\mathrm{C}$ cells that were injected into the yolk sac. Images are shown for each treatment condition on day 1 post-implantation (d1; start of treatment) and day 3 post-implantation (d3; end of experiment). Lower: waterfall plot displaying the change in tumor size (\%) from baseline (day $1=$ start of treatment) to day 3 after implantation for each individual xenograft. Zebrafish embryos were treated with a tenfold higher concentrations as substances are applied to the surrounding water and, as the estimated extent of compound absorption by the zebrafish larvae is one-tenth to one-twentieth of the cell culture treatment concentration. Solvent: DMSO, gray; HDAC8i: $100 \mu \mathrm{M} 20 \mathrm{a}$, green; crizotinib: $8 \mu \mathrm{M}$, blue; combination: $100 \mu \mathrm{M} 20 \mathrm{a}$ and $8 \mu \mathrm{M}$ crizotinib (orange). a-e Means from at least three independent experiments are shown, and the error bars represent SEM. ${ }^{*} p<0.05$; $* * p<0.01 ; * * * p<0.001$

cell death rate in non-neuroblastoma and non-ALK expressing cell lines (RD and VH7) was below 10\% for all treatment conditions (Fig. 5f), crizotinib treatment increased the amount of dead cells, presumably by interacting with c-MET (Fig. 5a). Altogether, simultaneous inhibition of ALK and HDAC8 eliminates ALK wild-type, constitutively active (F1174L-mutated and ALK-amplified), and $M Y C N$-amplified neuroblastoma cell lines, but not other malignant and non-transformed cell lines, and $M Y C N$ amplification sensitizes neuroblastoma cells to the combination treatment.

\section{Identification of RTK-ERK signaling pathways in the sensitization to HDAC8 targeting}

To mechanistically understand the interplay of HDAC8 inhibition and crizotinib, we next investigated the treatment effects on ALK activation and postulated downstream pathways (STAT3, PI3K-AKT and MAPK-ERK) [31, 36]. The activation of ALK by Y1604 phosphorylation, was not affected by HDAC8 inhibitor treatment (Supplementary Figure 4). To achieve comparable levels of ALK inhibition, SK-N-BE(2)-C cells were treated with $0.6 \mu \mathrm{M}$ and NB-1 cells with $0.05 \mu \mathrm{M}$ crizotinib (Supplementary Figure 4).

Single treatment of SK-N-BE(2)-C (ALK-wt), Kelly (F1174L), NB-1 (ALK-amp) and LAN-5 (R1275Q) cells with the HDAC8 inhibitor PCI-34051 had no effect on Y705 phosphorylation of STAT3, and had no significant effect on ERK1/2 phosphorylation, but slightly enhanced Y473 phosphorylation of AKT (Fig. 6a, b). In contrast, crizotinib treatment of SK-N-BE(2)-C, Kelly, NB-1 and LAN-5 cells inhibited phosphorylation of STAT3 (Kelly, NB-1) and, to a much higher degree, phosphorylation of ERK1/2, with the strongest inhibitory effect observed in NB-1 cells. The combination of both compounds reversed the HDAC8 inhibitor-mediated effects on AKT phosphorylation and abolished ERK1/2 phosphorylation in all four cell lines with complete abrogation of ERK signaling in NB-1 cells (Fig. 6a, b). The phosphorylation of AKT by HDAC8 inhibitor treatment is in line with the results of the initial RNAi screen, which indicated that the PI3K pathway mediates HDAC8 inhibitor anti-neuroblastoma effects. This PI3K pathway activation is also reflected by enhanced phosphorylation of the AKT downstream target mTOR and phosphorylation of the mTOR substrate S6K (Fig. 6c). Overall, these results confirm the relevance of the PI3KAKT-mTOR pathway in HDAC8 inhibitor-mediated effects, and ALK inhibition by crizotinib abolished the effect of the HDAC8 inhibitor on AKT. However, inhibition of ALK by crizotinib affects the ERK pathway, and treatment with the HDAC8 inhibitor potentiates inhibition of the ERK pathway.

\section{Inhibition of RTK-mediated signaling shifts the HDAC8 inhibitor-mediated phenotype from differentiation to cell death}

To further characterize the role of PI3K pathway activity in HDAC8 inhibitor-mediated effects, we treated SK-N-BE (2)-C cells with PCI-34051, the PI3K inhibitor LY294002, the mTOR inhibitor rapamycin or the dual $\mathrm{PI} 3 \mathrm{~K} / \mathrm{mTOR}$ inhibitor BEZ235. Co-treatment blocked the HDAC8 inhibitor-induced CDKN1 mRNA upregulation (Fig. 7a, b; Supplementary Figure 5A-C), increased the amount of cells surviving long-term HDAC8 inhibitor treatment (Fig. 7c) and reduced the outgrowth of neurofilament-positive structures (Fig. 7d; Supplementary Figure 5D). This indicates that the PI3K-AKT-mTOR axis is relevant for the differentiating phenotype induced by HDAC8 inhibitor treatment of SK-N-BE(2)-C cells, rendering the combination of $\mathrm{PI} 3 \mathrm{~K} / \mathrm{mTOR}$ inhibitors with HDAC8 inhibitors counterproductive. As recent studies showed that HDAC inhibitors act in cooperation with PI3K and mTOR 
inhibitors to inhibit tumor growth in MYC-driven tumors [37, 38], we tested the HDAC class I inhibitor entinostat (MS-275) at a concentration ineffective for HDAC8 (500 nM) [39] and combined it with the PI3K or mTOR inhibitor. Indeed, both LY294002 and rapamycin significantly enhanced entinostat-mediated effects on cell viability
(Supplementary Figure 5E, F), suggesting that HDAC1-3 inhibitors, but not HDAC8 inhibitors, cooperate with PI3K/ mTOR inhibitors in our neuroblastoma MYCN-driven model.

As ALK inhibition by crizotinib abolished the effect of the HDAC8 inhibitor on AKT but strongly inhibited ERK,
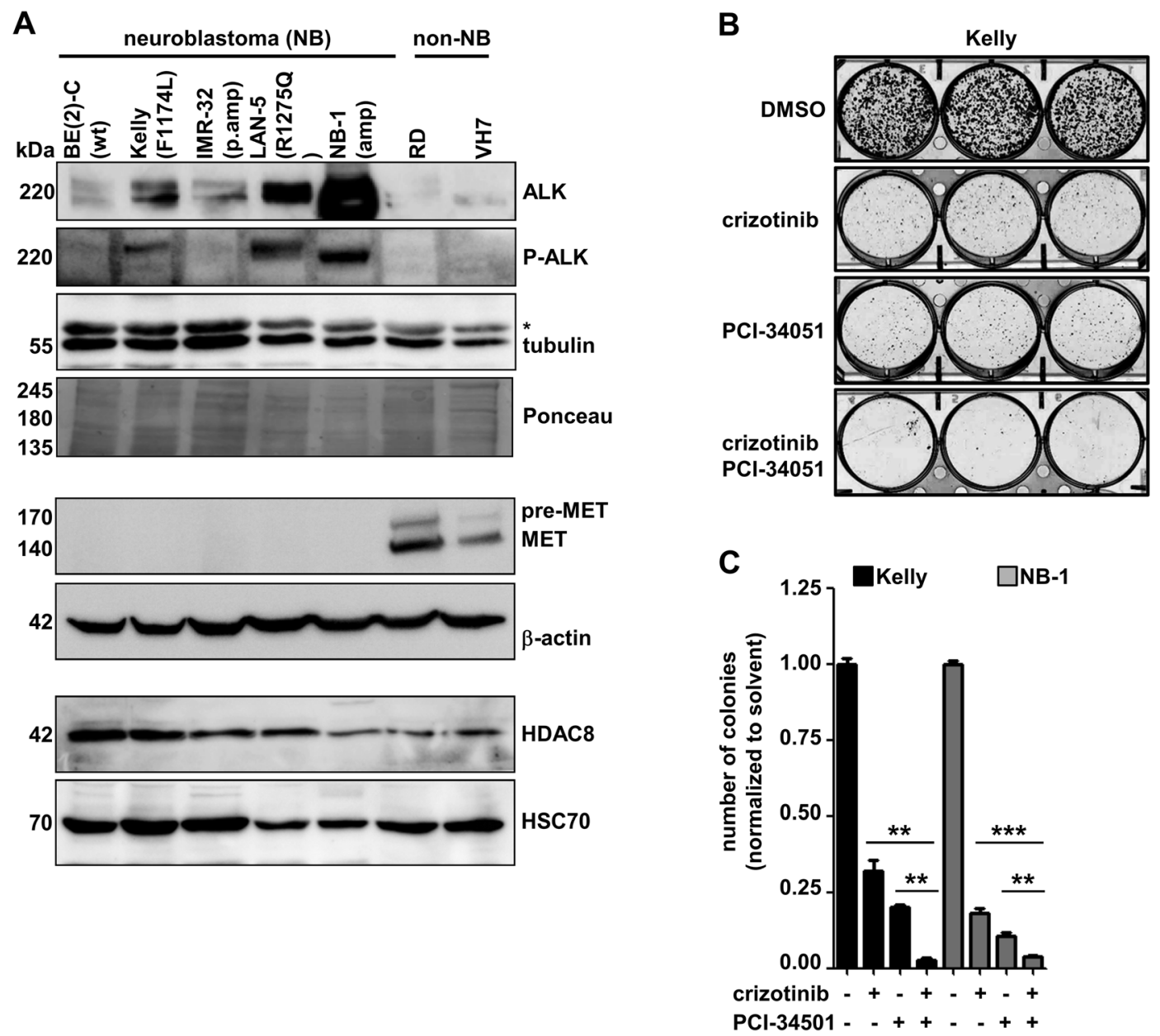

D

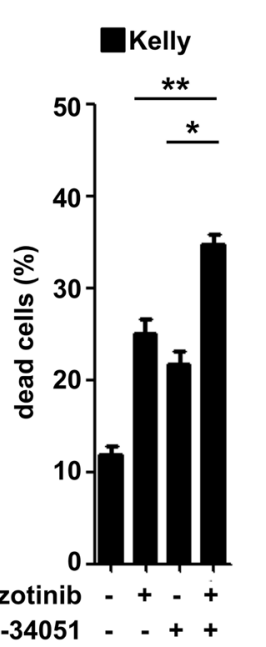

E
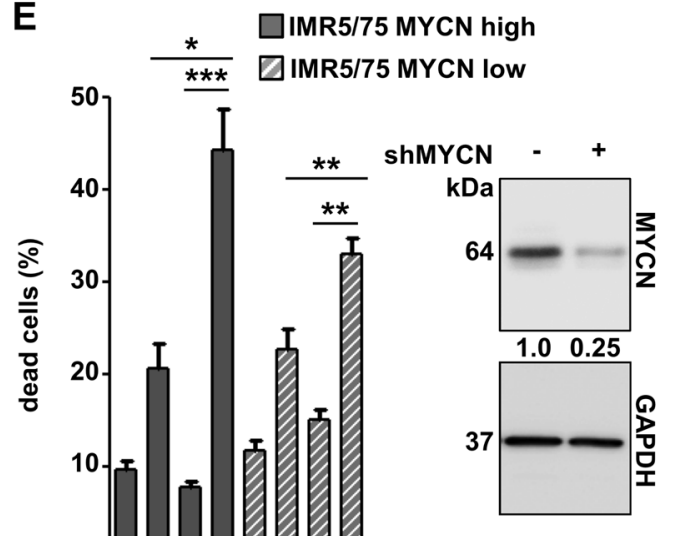

$\mathbf{F}$

중 RD GVH7 
Fig. 5 Dual targeting of ALK and HDAC8 efficiently targets neuroblastoma cell lines. a Expression of ALK, P-ALK, MET and HDAC8 protein levels in a panel of neuroblastoma (NB) and nonneuroblastoma cell lines. Tubulin, actin and HSC70 as well as Ponceau staining of the membrane served as a loading control. *Unspecific band (b) Kelly cells (5000 cells/well) were treated with crizotinib (0.8 $\mu \mathrm{M})$ alone or in combination with PCI-34051 $(6 \mu \mathrm{M})$, and colonies were stained after 10 days. $\mathbf{c}$ Bar diagram displaying the quantification of colonies upon treatment of Kelly and NB-1 cell lines with crizotinib $(0.8 \mu \mathrm{M}$ for Kelly, $0.05 \mu \mathrm{M}$ for NB-1) or PCI-34051 ( $6 \mu \mathrm{M}$ for Kelly, $4 \mu \mathrm{M}$ For NB-1) alone or in combination. d Kelly cells were treated with crizotinib $(0.8 \mu \mathrm{M})$ alone or in combination with PCI-34051 (6 $\mu \mathrm{M})$ and then monitored for $96 \mathrm{~h}$ after treatment for cell death using trypan blue staining (dead cells: trypan blue-positive cells). e IMR5/75 cells containing the tetracycline-inducible system for shRNA-mediated knockdown of MYCN were treated with crizotinib $(0.4 \mu \mathrm{M})$ alone or in combination with PCI-34051 $(3 \mu \mathrm{M})$ and then monitored for $96 \mathrm{~h}$ after treatment for cell death using trypan blue staining (dead cells: trypan blue-positive cells). Cells were treated with $1 \mu \mathrm{g} / \mathrm{ml}$ doxycycline ( $S h M Y C N)$ or solvent control (off) 2 weeks prior to compound treatment. Right: Expression of MYCN protein upon induction of knockdown with doxycycline $(+)$ for two weeks. GAPDH served as a loading control. Numbers indicate MYCN expression normalized to GAPDH expression. f Non-neuroblastoma cell lines RD and VH7 were treated with crizotinib $(0.8 \mu \mathrm{M})$ alone or in combination with PCI-34051 $(6 \mu \mathrm{M})$ and then monitored for $96 \mathrm{~h}$ after treatment for cell death using trypan blue staining (dead cells: trypan blue-positive cells). c-f Means from at least three independent experiments are shown, and the error bars represent SEM. $* p<0.05 ; * * p<0.01$; ***p $<0.001$

which resulted in enhanced cell death, we hypothesized that RTK inhibition shifts the HDAC8 inhibitor-mediated differentiation phenotype toward neuroblastoma cell death. One characteristic marker of the HDAC8 inhibitor phenotype is up-regulation of NTRK1 [9], especially upon cotreatment with the differentiation-inducing agent retinoic acid (ATRA) [10]. The combined treatment of SK-N-BE (2)-C cells with PCI-34051 and crizotinib not only blocked HDAC8 inhibitor-induced upregulation of NTRK1 but also diminished the powerful effect of the PCI-34051/ATRA combination (Fig. 7e). Finally, we asked whether there was an overlap between the published ALK inhibitor gene expression signature [40] and HDAC8 inhibitor-mediated gene expression effects. A subset of ALK signature genes was affected by HDAC8 inhibitor treatment. Seven genes were regulated in the opposite direction (e.g. MAPK negative feedback regulator SPRY4) and two genes (RET, $V I P$ ) were regulated in the same direction (Supplementary Figure 5G, Supplementary Table 4). The proto-oncogene and neuronal marker RET has been proposed as a drug target in the context of aberrant ALK activation and its overexpression is driven by mutant ALK [40]. Realtime PCR analysis confirmed downregulation of RET in ALK wildtype SK-N-BE(2)-C cells upon treatment with PCI34051 (Fig. 7f). In summary, we conclude that targeting ALK diminishes HDAC8 inhibitor treatment-mediated neuroblastoma cell differentiation and cell cycle arrest and inhibits ERK signaling, shifting the phenotype toward efficient neuroblastoma cell death and HDAC8 inhibition affects expression of ALK targets, such as RET (model Fig. $7 \mathrm{~g}$ ).

\section{Discussion}

The use of HDAC inhibitors, which are most commonly utilized as broad-spectrum agents inhibiting the activity of multiple HDAC isotypes, is emerging as an effective cancer treatment strategy [41, 42], and several inhibitors are in phase I-III clinical trials. However, simultaneous inhibition of several HDAC family members confers greater toxicity, resulting in dose-limiting side effects that restrict the anticancer potential of these inhibitors. Using HDAC8-dependent neuroblastoma tumor models, we previously demonstrated that inhibition of a single HDAC isotype is more effective and less toxic than unspecific HDAC inhibition [10]. In comparison to the very sensitive cell line IMR-32, responding to the treatment by cell death, some neuroblastoma cell lines, such as SK-N-BE(2)-C, respond with a differentiation-like phenotype characterized by cell cycle arrest and outgrowth of neurite-like extensions.

\section{ALK activity and downstream pathways}

Here, we identified the ALK pathway as an HDAC8 inhibitor resistance pathway. ALK-activating point mutations predominate in neuroblastoma [43, 44], making it an attractive therapeutic target. ALK mutations, e.g., germline ALK R1275Q, enable the constitutive activation of the kinase domain, which drives tumor cell malignancy and in vivo tumorigenicity $[45,46]$. Studies in neuroblastoma have revealed that R1275Q and wild-type ALK-amplified cell lines are highly sensitive to crizotinib [44, 47, 48], whereas cell lines harboring the ALK F1174L mutation are less so, but still more responsive to treatment than nonamplified, wild-type ALK cell lines [44, 47]. The F1174L mutation bears strong oncogenic capacity and correlates with $M Y C N$ amplification, potentiating the oncogenic activity of MYCN in neuroblastoma, and is linked to acquired resistance to crizotinib [30, 49]. Moreover, mutant $A L K$ drives the expression of the tyrosine kinase RET, which is a sympathetic neuronal marker of the cholinergic lineage, but also a proto-oncogene and promising drug target in the context of aberrant ALK activation [40]. In line with the results of Lambertz et al. ALK inhibition alone did not affect RET expression in the ALK wildtype cells, whereas the combination of ALK and HDAC8 inhibitors decreased RET expression in our study. Inhibition of 
Fig. 6 HDAC 8 and ALK converge at the level of ALK downstream targets. a Detection of phosphorylation levels of STAT3, AKT and ERK1/2 in neuroblastoma cells treated for $6 \mathrm{~h}$ with crizotinib $(0.6 \mu \mathrm{M}$ SKN-BE(2)-C, Kelly and LAN-5, $0.05 \mu \mathrm{M}$ NB-1) alone or in combination with PCI-34051 (6 $\mu \mathrm{M}$ SK-N-BE(2)-C, Kelly, NB-1 and $3 \mu \mathrm{M}$ LAN-5). Numbers in the upper row indicate pSTAT3 levels normalized to STAT3 expression, numbers in the middle row indicate $\mathrm{pAKT}$ levels normalized to AKT expression, and numbers in the lower row indicate $\mathrm{pERK} 1 / 2$ levels normalized to ERK1/2 expression. Lower: western blot analysis showing acetylation levels of HDAC8 substrate SMC3 in neuroblastoma cells. Actin served as a loading control. b The bar diagrams display the quantification of at least three independent experiments from (a). Means from at least three independent experiments are shown, and the error bars represent SEM. $* p<$ $0.05 ; * * p<0.01 ; * * * p<0.001$. A one-sample $t$-test was used to test whether means were significantly different from a hypothetical value (1.0). c Detection of phosphorylation levels of mTOR and S6K1 in SK-N-BE(2)-C cells treated for $2 \mathrm{~h}$ with PCI-34051 (PCI, $6 \mu \mathrm{M}$ ) alone or in combination with rapamycin (RAPA, $100 \mathrm{nM}$ ). Actin served as a loading control

\section{A}
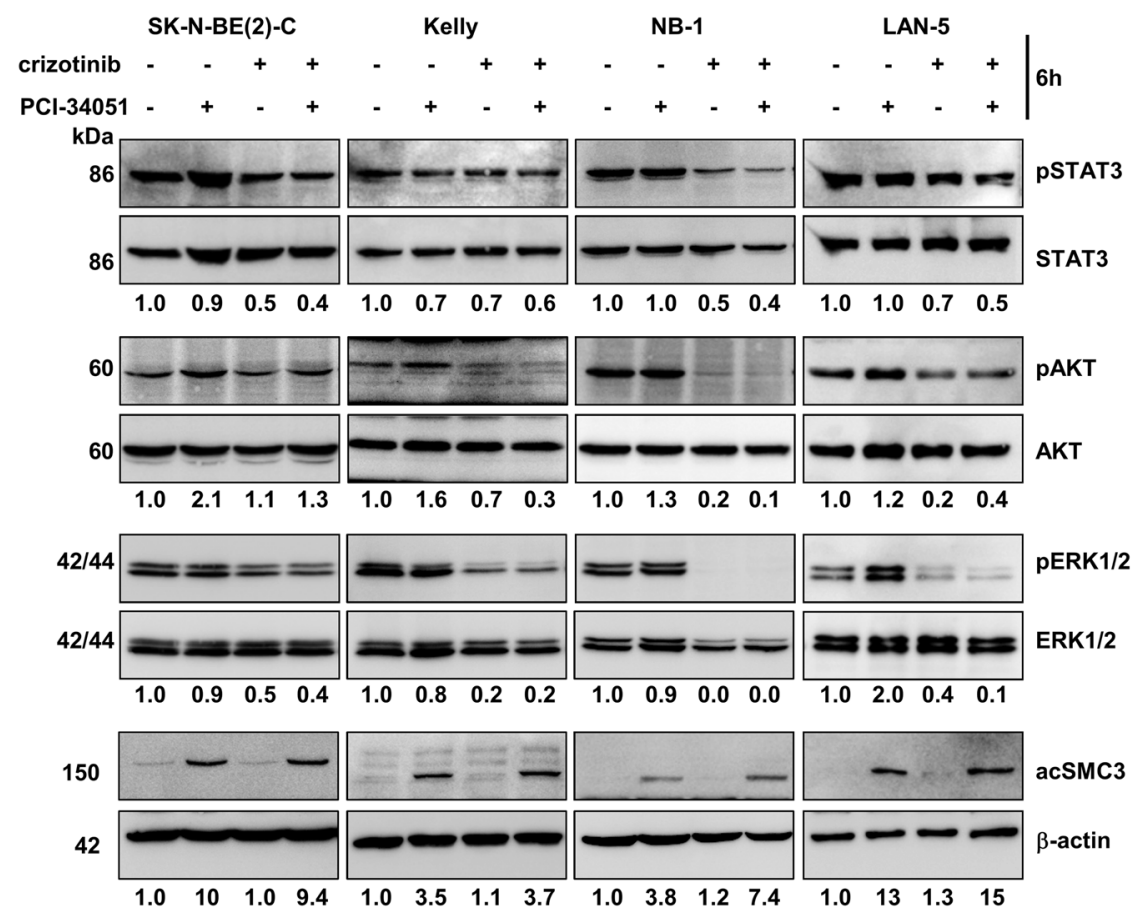

B
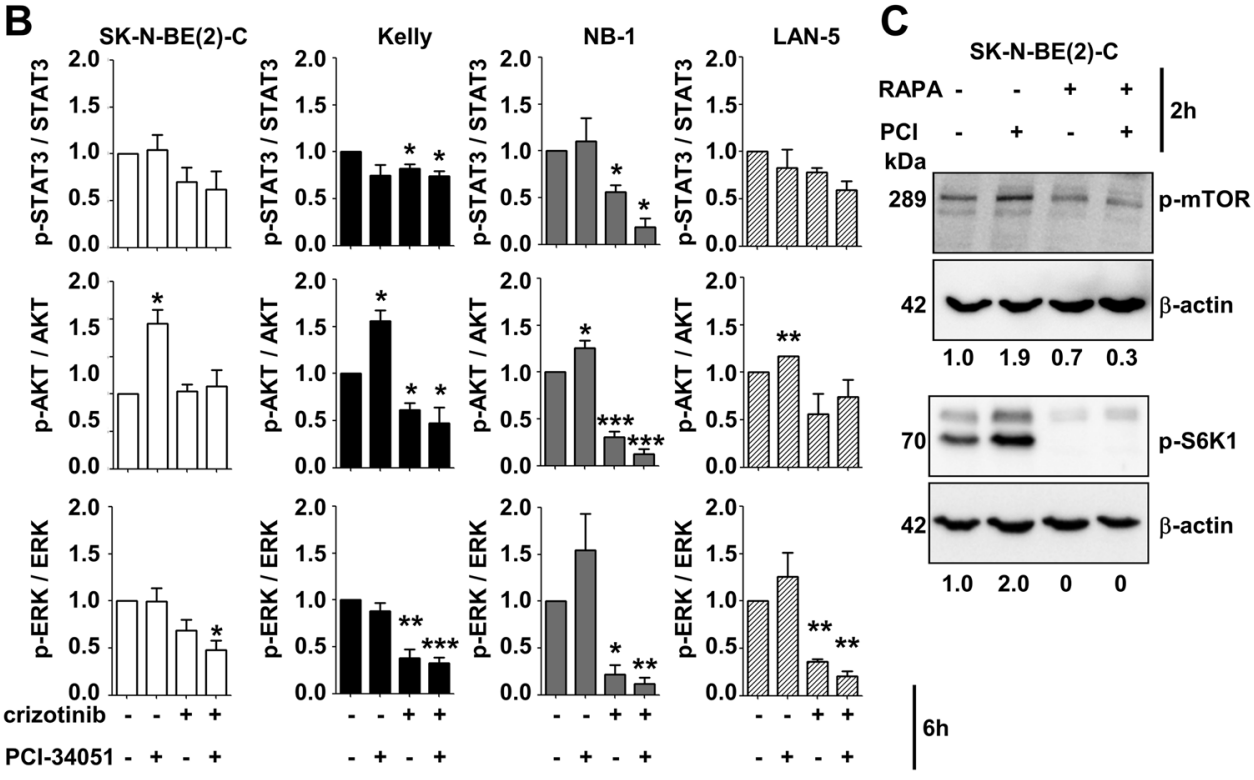

HDAC8 counteracted the downregulation of MAPK negative feedback regulator SPRY4, which might explain the enhanced inhibitory effect of combination treatment on MAPK/ERK signaling.

ALK aberrations are found in 14\% (10\% mutations, 4\% amplification) of high-risk neuroblastoma patients, and are biomarkers of poor outcome [50]. In neuroblastoma, ALK is almost ubiquitously expressed on the cell surface and expression is restricted to tumor cells [51, 52]. Duijkers et al. [53] demonstrated that, while neuroblastoma cells often express ALK at high levels, the expression in mutated cell lines is even higher with superior responses to inhibition. In our study, all investigated cell lines responded to the combination treatment of crizotinib with the HDAC8 inhibitor, suggesting a sensitization effect independent of ALK mutation or ALK amplification. Of note, the combination treatment of crizotinib with the HDAC8 inhibitor affected neither untransformed cells, nor other embryonic cancer cell models. Our results support at least two mechanisms of action at the level of RTKs, inhibition of the MAPK/ERK pathway and/or induction of the PI3K/AKT/mTOR axis. Although HDACs directly regulate gene transcription, e.g., 
via histone deacetylation and as interaction partners in corepressor complexes, so far no HDAC8-containing corepressor complex has been identified [54]. In contrast, many non-histone substrates have been described, such as SMC3 [55], ARID1A [56], and nuclear receptor ERRalpha [57].
The list of potential HDAC8 substrates increased massively after the description of the human acetylome by Choudhary et al. [58]. Hence, future studies will elucidate whether indirect effects mediated by one of the potential HDAC8 substrates alter PI3K signaling in neuroblastoma.

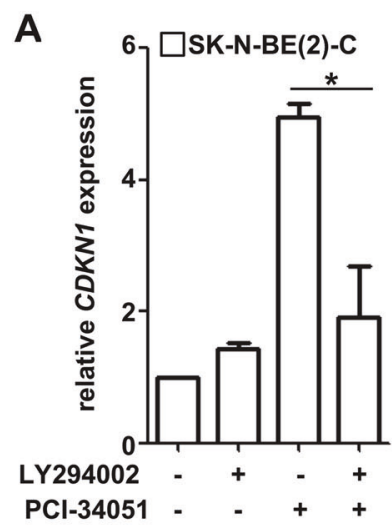

D

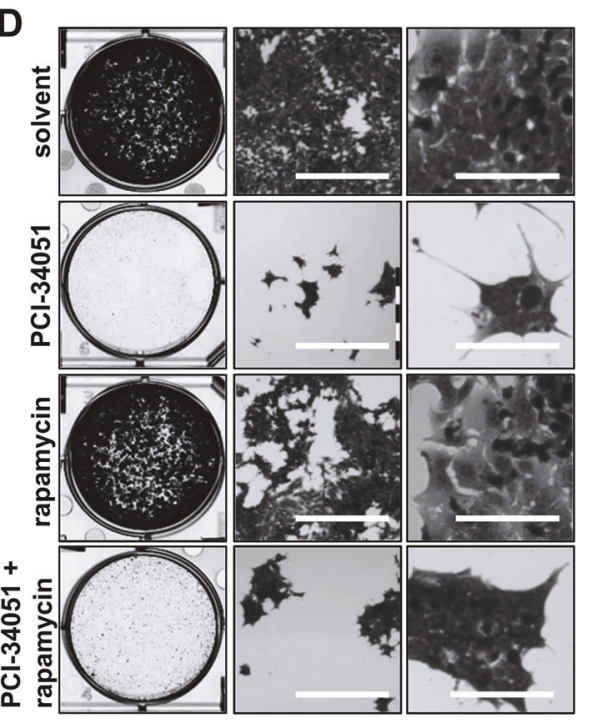

B

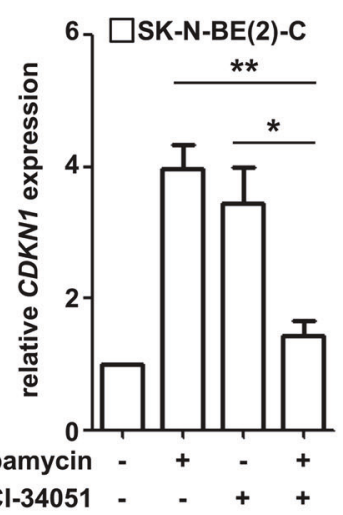

E

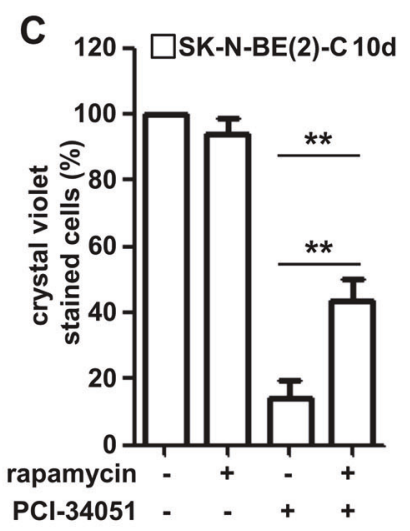

$\mathbf{F}$
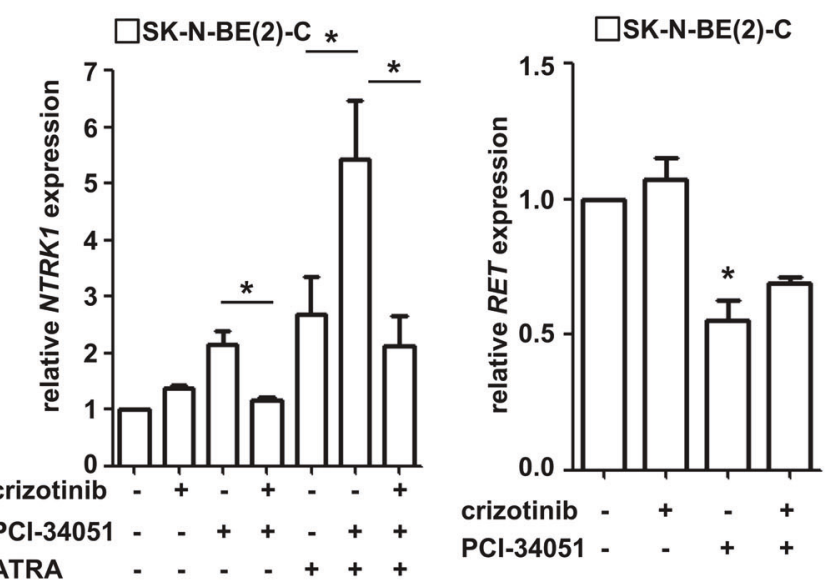

G

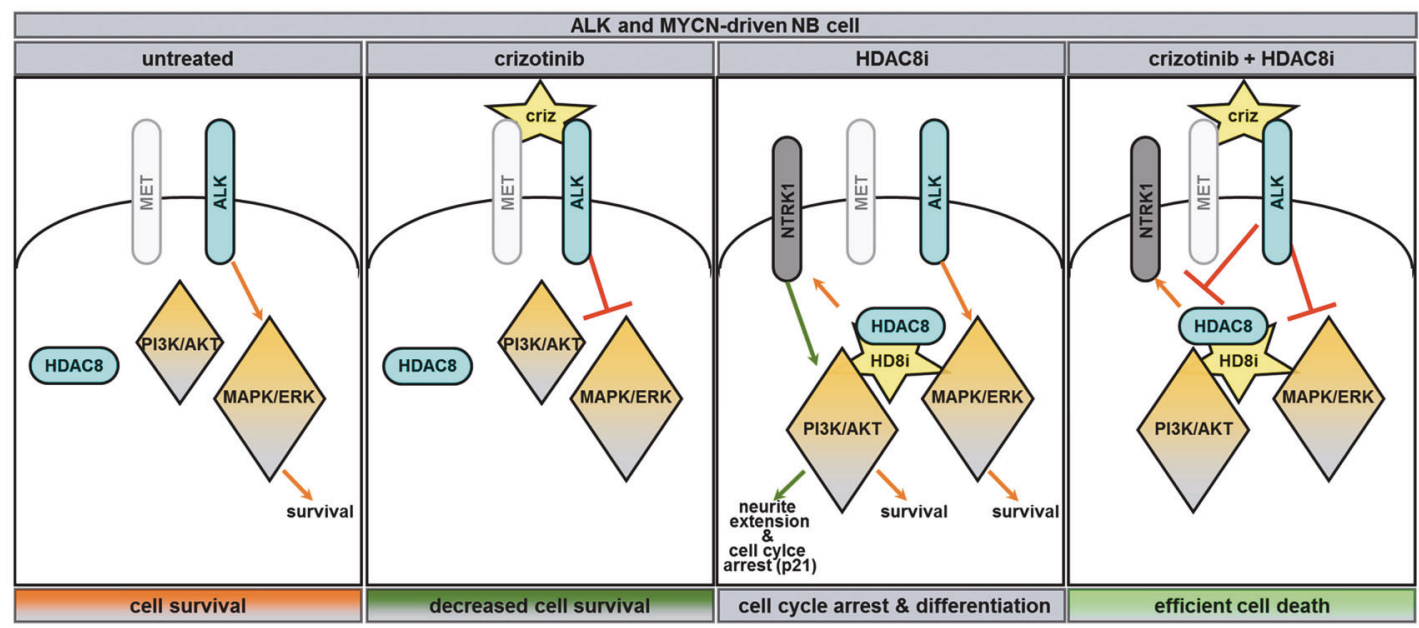


Fig. 7 RTK inhibition shifts HDAC8 inhibitor phenotype from differentiation to cell death. a SK-N-BE(2)-C cells were treated with LY294002 $(10 \mu \mathrm{M})$ alone or in combination with PCI-34051 $(6 \mu \mathrm{M})$, and RNA was isolated $72 \mathrm{~h}$ after treatment for real-time PCR analysis of $C D K N 1$ (p21) expression. b SK-N-BE(2)-C cells were treated with rapamycin $(100 \mathrm{nM})$ alone or in combination with PCI-34051 $(6 \mu \mathrm{M})$, and RNA was isolated $72 \mathrm{~h}$ after treatment for real-time PCR analysis of $C D K N 1$ (p21) expression. c The bar diagram displays the ImageJbased quantification of crystal violet-stained SK-N-BE(2)-C cells 10 days after treatment with rapamycin $(100 \mathrm{nM})$ alone or in combination with PCI-34051 $(6 \mu \mathrm{M})$. Data were normalized to solvent (DMSO)-treated cells. d Representative pictures of crystal violetstained SK-N-BE(2)-C cells 10 days after treatment with rapamycin $(100 \mathrm{nM})$ alone or in combination with PCI-34051 $(6 \mu \mathrm{M})$. Scale bars, middle column: $500 \mu \mathrm{m}$; scale bars, right column: $100 \mu \mathrm{m}$. e SK-N-BE (2)-C cells were treated with crizotinib $(0.8 \mu \mathrm{M})$ alone or in combination with PCI-34051 $(6 \mu \mathrm{M})$. Where indicated, treatment was additionally combined with ATRA $(10 \mu \mathrm{M})$. RNA was isolated $72 \mathrm{~h}$ after treatment for real-time PCR analysis of NTRK1 expression. f SK-N-BE (2)-C cells were treated with crizotinib $(0.8 \mu \mathrm{M})$ alone or in combination with PCI-34051 $(6 \mu \mathrm{M})$, and RNA was isolated $72 \mathrm{~h}$ after treatment for real-time PCR analysis of RET expression. $\mathbf{g}$ Model of the lethal interactions of crizotinib with HDAC8 inhibitors. The specific effects of crizotinib on ALK downstream pathways (mainly via ERK inhibition) shift the rather mild HDAC8 inhibitor phenotype, characterized by cell cycle arrest and early signs of differentiation (neurite extension), toward effective neuroblastoma cell death. HD8i $=$ HDAC8i, HDAC8 inhibitor; criz, crizotinib. a-c, e, f Means from at least three independent experiments are shown, and the error bars represent SEM. * $p<0.05 ; * * p<0.01 ; * * * p<0.001$

Several studies have described a role of factor (e.g., IGF, NGF)-mediated activation of the PI3K/AKT/mTOR/S6K signaling pathway in peripheral nerve outgrowth and branching [59-64]. It is conceivable that activation of this pathway mediates the HDAC8 inhibitor-induced priming of neuroblastoma cells for neurite extension and branching [10].

\section{Conclusion}

We have shown that HDAC8 and ALK pathways converge at downstream nodes, and that simultaneous inhibition of HDAC8 and the RTK-ERK pathway leads to efficient cell death of neuroblastoma cells (model Fig. $7 \mathrm{~g}$ ). Altogether, our results provide a solid rationale for the combination treatment of HDAC inhibitors with TKIs, such as crizotinib, which shifts cell cycle arrest and the differentiation phenotype toward effective tumor cell death.

\section{Methods}

\section{Cell culture, transfections, and reagents}

All cell lines were grown under standard conditions as described previously [65]. Human neuroblastoma cell lines SK-N-BE(2)-C (European Collection of Authenticated Cell Cultures (ECACC), Salisbury, UK), IMR-32 (DSMZ, Braunschweig, Germany), Kelly (DSMZ), NB-1 (\#RCB1953, RIKEN cell bank, Japan), LAN-5 (generously provided by L. Savelyeva, laboratory of F. Westermann, DKFZ, Germany) and tetracycline-inducible shMYCN IMR5/75 [66] (generously provided by the laboratory of F. Westermann), were grown under standard conditions in Dulbecco's modified Eagle's medium (DMEM) with L-glutamine, $4.5 \mathrm{~g} / 1$ glucose (Lonza, Basel, Switzerland) and $1 \%$ non-essential amino acids (NEAA) (Invitrogen, Darmstadt, Germany) or in RPMI1640 with L-glutamine (Lonza) and 1\% NEAA. All media were supplemented with $10 \%$ fetal bovine serum (FBS) (Sigma, Munich, Germany). The embryonal rhabdomyosarcoma cell line RD (kindly provided by S. Fulda, University of Frankfurt, Germany) was grown in DMEM plus GlutaMAX ${ }^{\mathrm{TM}}$-I supplemented with $10 \%$ FBS. Nontransformed, proliferatively active primary human foreskin fibroblasts from an infant donor (VH7) were a gift from Petra Boukamp, German Cancer Research Center (DKFZ), Heidelberg, Germany. Fibroblasts were maintained in DMEM/Ham's F12 (Invitrogen) supplemented with $10 \%$ FBS and 1\% NEAA. All cell lines were genotyped and routinely tested for mycoplasma contamination. The ALK status of neuroblastoma cell lines was confirmed by sequencing (SK-N$\mathrm{BE}(2)-\mathrm{C}, \mathrm{Kelly})$ and compared to the literature [53, 67]: SK-N-BE(2)-C: wild type. IMR-32: partial ALK amplification [67]. NB-1: ALK-amplified. LAN-5: ALK R1275Q mutated. Kelly: ALK F1174L mutated. Transient transfections were performed as described previously [9]. The final RNAi concentration was $20 \mathrm{nM}$ for all experiments. All RNAi-IDs used in the screen are listed in Supplementary Table 1 (Dharmacon). In addition, HDAC8 (siRNA1, ID 120597 and siRNA2, ID 120599, Ambion, Huntingdon, UK), ALK (siRNA3, D-003103-07 and siRNA4, D-003103-09 Dharmacon, GE Healthcare) siRNAs and the corresponding negative control siRNA (Silencer Negative Control 1, Silencer Negative Control 5; Ambion and RLuc Duplex (Dharmacon) were used.

\section{Reagents}

HDAC8-selective inhibitors Cpd2 [13] (stock concentration $250 \mathrm{mM}$ ) and PCI-34051 [14] (stock $20 \mathrm{mM}$; Pharmacyclics Inc., Sunnyvale, CA, USA) were dissolved in DMSO. Crizotinib (PF-02341066, stock $10 \mathrm{mM}$ in DMSO, Selleckchem, Houston, USA) and LDK378 (stock $10 \mathrm{mM}$ in DMSO, Selleckchem, Houston, USA) were used as ALK inhibitors. Erlotinib (stock $20 \mathrm{mM}$; Focus Biomolecules), rapamycin (stock $1 \mathrm{mM}$; Sigma), LY294002 (stock 
$10 \mathrm{mM}$, Cayman) and 20a (Prof. Sippl; University of Halle, Germany) were dissolved in DMSO. EGF stock at $25 \mu \mathrm{g} / \mathrm{ml}$ (PromoKine, Heidelberg, Germany) was also used.

\section{Kinome-wide siRNA screen}

The RNAi screen was performed in duplicate with siRNA-coated 384-well plates using a pooled siRNA library (Dharmacon SMART pool library; Thermo Scientific), where each kinase gene was targeted by a pool of four single siRNAs (Supplementary Table 5). The transfection mix for a single well contained $5 \mu \mathrm{l}$ siRNApool, $0.05 \mu$ l Dharmafect and $14.95 \mu$ l RPMI. SK-N$\mathrm{BE}(2)-\mathrm{C}$ cells $(800$ cells/well) were seeded on top, and the final siRNA concentration was $25 \mathrm{nM}$. Plates were incubated for $24 \mathrm{~h}$. Plates were then treated for $96 \mathrm{~h}$ with either HDAC8 inhibitors or solvent (DMSO) control. CTG assays (Promega) were performed to measure cell viability. Medium was removed with a 24-channel comb, and $20 \mu \mathrm{l}$ of 1:4 diluted CTG reagent was added for $20 \mathrm{~min}$. Luminescence was measured with a Mithras LB940 plate reader (Berthold Technologies). Data were analyzed with web-cellHTS2 [68]. Comparisons of the duplicate experiments revealed high reproducibility of the screen (Supplementary Figure 1A). Positive controls targeting COPB2 (\#1), UBC (\#2) and PLK1 (\#3) and the negative control (Renilla Luciferase) siRNAs were used on each plate to monitor and compare the screen performance of all 18 plates (Supplementary Figure 1B). Mean $z$-factor (all plates): 0.34. Plate-wise $z$-factors (negative control: untreated; positive control: SAHA): 83_1 (Cpd2): 0.54; 83_2 (Cpd2): 0.50; 83_3 (Cpd2): 0.41; 84_1 (Cpd2): 0.19; 84_2 (Cpd2): 0.30; 84_3 (Cpd2): 0.19; 85_1 (PCI-34051): 0.34; 85_2 (PCI34051): 0.33; 85_3 (PCI-34051): 0.44; 86_1 (PCI34051): 0.35; 86_2 (PCI-34051): 0.33; 86_3 (PCI34051): 0.37; 87_1 (DMSO): 0.30; 87_2 (DMSO): 0.27; 87_3 (DMSO): 0.26; 88_1 (DMSO): 0.24; 88_2 (DMSO): 0.37; 88_3 (DMSO): 0.41. Supplementary Figure 1C displays the effect of all non-transfected control treatments, representing relative luminescence units compared with untreated cells, HDAC8 inhibitor (\#1 and \#2)-treated cells and solvent-treated non-transfected cells. To identify hits that significantly increase ("lethal hits") or decrease ("rescue hits") HDAC8imediated toxicity, we performed normalization for each treatment. The calculated treatment factor ( $t$-factor) was $1.930+/-0.07$ for HDAC8i \#1, $3.789+/-0.25$ for HDAC8i \#2 and $0.959+/-0.04$ for DMSO. Supplementary Figure 1D displays the data after normalization with the respective factor. A hit was defined as the $t$ factor HDAC8i-corrected $\mathrm{t}$-factor minus the DMSO- corrected $t$-factor $>60,000$ RLU (=rescue hit) or $<$ $-60,000 \mathrm{RLU}$ (=lethality hit). As an additional control, we performed a re-screen with selected hits targeted by single siRNAs in 384-well plates and also validated selected single siRNAs in 96-well plates.

\section{Cell counting, cell viability, cell death, and colony assay}

Cells were collected, pooled with the corresponding supernatant, centrifuged and resuspended in $1.5 \mathrm{ml}$ of cell culture media. Cell counts as well as cell viability were measured by automated trypan blue staining with a Vi-Cell XR Cell Viability Analyzer from Beckman Coulter (Krefeld, Germany). A caspase-3-like protease activity assay was performed as described previously [9]. Apoptosis quantification of propidium iodide-stained and ethanol-fixed cells by flow cytometry was performed as described previously [69]. Colony assay: In six-well plates, 500 cells were seeded and treated for $18-24 \mathrm{~h}$ after seeding, as indicated. After $96 \mathrm{~h}$ of treatment, the medium was changed to fresh untreated medium allowing the formation of colonies. After a minimum of another seven days, viable colonies were stained with crystal violet ( $1 \%$ in $70 \%$ ethanol). For quantification, the plates were scanned, and colonies were counted in 16-bit binary pictures with the ITCN plugin for ImageJ software (U. S. National Institutes of Health, Bethesda, MD, USA; http:// imagej.nih.gov/ij/).

\section{Western blot analysis}

Total cell lysates were obtained with NP-40 lysis buffer ( $150 \mathrm{mM}$ sodium chloride, $1.0 \% \mathrm{NP}-40,50 \mathrm{mM}$ Tris, $\mathrm{pH}$ 8.0), supplemented with protease inhibitor cocktail (cOmplete mini, Roche) and phosphatase inhibitors (PhosSTOP, Roche). Protein samples were denatured with 2mercaptoethanol at $95{ }^{\circ} \mathrm{C}$ for $5 \mathrm{~min}$. The following antibodies were used for detection: anti-pALK Y1604 (Cell Signaling Technology), anti-ALK (Cell Signaling Technology), anti-pSTAT3Y705 (Cell Signaling Technology), anti-STAT3 (Cell Signaling Technology), anti-pERK1/2 (Cell Signaling Technology), anti-ERK1/2 (Cell Signaling Technology), anti-pAkt Y473 (Cell Signaling Technology), anti-AKT, anti-human PARP (Santa Cruz Biotechnology), anti-HDAC8 (H-145;polyclonal; Santa Cruz, Santa Cruz, CA, USA), anti-p-mTOR (Ser2448; Upstate), anti-p-S6K1 (Thr412; Upstate), anti-MET (Cell Signaling Technology), anti-MYCN (Santa Cruz), anti-ac-SMC3 (provided by Prof. K Shirahige, University of Tokyo, Tokyo, Japan) [55], antiHSC70 (Santa Cruz), anti- $\beta$-actin (clone AC-15; Sigma), anti-actinin (H-2; Santa Cruz) and anti-GAPDH (clone 6C5; Merck). 
Real-time, reverse-transcription polymerase chain reaction

Real-time polymerase chain reaction (PCR) was performed as described previously [9]. Data were normalized against the housekeeping genes SDHA and HPRT [70] and set in relation to a negative control. The following specific primer pairs were used: CDKN1A (p21WAF1/CIP1) (forward: 5'TGG AGA CTC TCA GGG TCG AAA-3', reverse: 5'GGC GTT TGG AGT GGT AGA AAT C-3'), HPRT (forward: 5'-TGA CAC TGG CAA AAC AAT GCA-3', reverse: 5'-GGT CCT TTT CAC CAG CAA GCT-3'), NTRK1 (forward: 5'-CAG CCG GCA CCG TCT CT-3', reverse: 5'-TCC AGG AAC TCA GTG AAG ATG AAG3'), SDHA (forward: 5'-TGGGAACAAGAGGGCATCTG3', reverse: 5'-CCACCACTGCATCAAATTCATG-3').

\section{Immunofluorescence and 4',6-diamidino-2- phenylindole staining}

SK-N-BE(2)-C cells $\left(3 \times 10^{4}\right)$ were grown on 8 -well chambers (ibidi). Six days after treatment with compounds, cells were fixed for $15 \mathrm{~min}$ in $2 \%(\mathrm{w} / \mathrm{v})$ paraformaldehyde and permeabilized for 15 min with $0.1 \%$ (v/v) Triton X-100 in PBS. After washing thrice with PBS, cells were blocked [PBS-Triton with $10 \%$ goat serum and $0.25 \%$ $(\mathrm{w} / \mathrm{v}) \mathrm{BSA}$ ] for $1 \mathrm{~h}$ at room temperature, incubated overnight with anti-NEF-M antibody (polyclonal rabbit, Millipore; $1: 500$ ) at $4{ }^{\circ} \mathrm{C}$, washed with PBS, and incubated with Cy3-labeled goat anti-rabbit antibody (Dianova; 1:200) for $3 \mathrm{~h}$ at room temperature. Nuclei were co-stained with 250 $\mathrm{ng} / \mathrm{mL}$ 4',6-diamidino-2-phenylindole (DAPI) solution and analyzed with an Olympus CKX41 microscope and ColorView I FW camera.

\section{Statistical analysis}

All cell culture experiments were performed in duplicate or triplicate, and each experiment was repeated at least three times. A two-tailed $t$-test, unpaired or paired where appropriate, was performed using GraphPad Prism Version 5.00 (GraphPad Software) to compare treatment groups. $p$-values of less than 0.05 were considered significant. The relationship between $H D A C 8$ and $A L K$ expression was determined using the Pearson productmoment correlation, where the $p$-value reflects the result of a $t$-test with the null hypothesis that the correlation between the variables is equal to zero. Kaplan-Meier curves were compared using a log-rank test, where the $p$ value reflects whether the difference between the survival curves is significant. The software program $R$ ( $R$ version 3.2.2, 2015; The R Foundation for Statistical Computing) was used to calculate correlation coefficients, identify differentially expressed genes, generate survival curves and perform associated calculations and Cox regression analyses.

\section{Gene expression analysis}

\section{Web-based}

R2 (R2: microarray analysis and visualization platform; http://r2.amc.nl) was used to investigate HDAC8 and ALK expression in publicly available cohorts of primary neuroblastoma patients. For the Academic Medical Center (AMC) cohort (Gene Expression Omnibus (GEO) database accession no. GSE16476), expression of HDAC8 was detected using the probeset 223345_at, and ALK expression was detected using the probeset 208211_s_at. Patient characteristics were published previously [26]. Expression data for the German Neuroblastoma cohort (GEO database accession no. GSE45547) was obtained from R2 using the probeset Ukv4_A_24_P254965 to detect HDAC8 expression and the probeset Ukv4_A_23_P324304 to detect ALK expression. Patient characteristics were published previously [27]. Current survival data for the German Neuroblastoma cohort were provided by $M$. Fischer (University of Cologne, Germany).

Gene ontology enrichment analysis was performed with GOrilla (http://cbl-gorilla.cs.technion.ac.il/), pathway analysis for the detection of significantly enriched signaling pathways was performed with REACTOME (http://www. reactome.org/), and a protein class analysis was performed based on the PANTHER classification system [15].

\section{Own data}

Total RNA was isolated from three independent neuroblastoma cell cultures samples, using the RNeasy MiniKit (Qiagen). For microarray analysis, $1 \mu \mathrm{g}$ RNA per sample was used. Gene expression analysis was performed at the house-internal Genomics and Proteomics Core Facility using human whole genome HT$12 \mathrm{v} 4$ BeadChips $^{\circledast}$. Normalization of the raw intensity data was performed by the microarray unit of the DKFZ Genomics and Proteomics Core Facility with Illumina BeadStudio Data Analysis Software version v4_r2. GEO number: GSE110817. Differentially regulated genes in SK-N-BE(2)-C cells after six days of treatment with PCI-30451 $4 \mu \mathrm{M}$ compared with solvent control were identified using the topTable function in the limma package in $\mathrm{R}$ [71]. A list of the most differentially expressed genes was created using the following fold change cutoffs: 1.5 -fold or more for upregulation and 0.67 -fold or less for downregulation. 


\section{Zebrafish lines}

Care and breeding of zebrafish were done under standardized conditions. Zebrafish wild-type TE line was raised at $28^{\circ} \mathrm{C}$. Embryos used for tumor injections were maintained in E3 buffer supplemented with $0.2 \mathrm{mM}$ 1-phenyl-2thiourea (PTU, Sigma).

\section{Cell preparation and xenotransplantation}

SK-N-BE(2)-C cells were cultured to $70-80 \%$ confluence, then washed once with PBS (Lonza, Basel, Switzerland), trypsinized (Gibco), counted and resuspended in phenol red-free Roswell Park Memorial Institute medium (RPMI, Gibco). Tumor cells were labeled by incubation with CellTracker CM-DiI (Thermo Fisher Scientific, Waltham, MA, USA) for $5 \mathrm{~min}$ at $37^{\circ} \mathrm{C}$, and then for an additional $15 \mathrm{~min}$ at $4{ }^{\circ} \mathrm{C}$. To minimize cell clumping, DNase I (250 Kunitz units/ml, Sigma) was added to the cell suspension. Following the incubation, cells were washed twice with $10 \%$ FCS RPMI, twice with serum-free RPMI and resuspended in serum-free RPMI to a final concentration of $1.0 \times 10^{8} \mathrm{cell} / \mathrm{ml}$. Before implantation, zebrafish were anesthetized with tricaine $(0.02 \%$, Sigma) and embedded in a lateral position in $1.0 \%$ low gelling temperature agarose (Sigma). Between 150 and $250 \mathrm{CM}$-DiI-labeled tumor cells were injected into the yolk sac of each zebrafish larva using FemtoJet express microinjector (Eppendorf, Hamburg, Germany) and glass microinjection needles (Science Products, Hofheim, Germany). Larvae were transferred to $34{ }^{\circ} \mathrm{C} 1 \mathrm{~h}$ after tumor cell injection.

\section{Drug treatment and efficiency evaluation}

Tumor xenografts were evaluated by fluorescence microscopy (Olympus, Hamburg, Germany) $2 \mathrm{~h}$ post implantation. Only larvae with red fluorescence at the injection site were used for drug testing. Selected embryos were transferred to 48-well uncoated plates (Corning) and incubated in freshly prepared E3 medium containing drugs or solvent. The medium was replaced daily. Tumor growth was evaluated by confocal microscopy before drug exposure as well as $48 \mathrm{~h}$ post treatment. For imaging, fish were anesthetized with tricaine $(0.02 \%$, Sigma) and embedded in a lateral position in $1.0 \%$ low gelling temperature agarose (Sigma) in chambered coverslips (ibidi, Martinsried, Germany). Images of living larvae were obtained using a Zeiss LSM 710 confocal microscope (Zeiss, Oberkochen, Germany) and ZEN software (Zeiss). Tumor progression was evaluated using Fiji software and a semi-automated macro. Zebrafish embryos were treated with a ten-fold higher concentration of crizotinib $(8 \mu \mathrm{M})$ and $20 \mathrm{a}(100 \mu \mathrm{M})$ as substances are applied to the surrounding water and, as the estimated extent of compound absorption by the zebrafish larvae is one-tenth to one-twentieth of the cell culture treatment concentration [28, 72].

Acknowledgements We thank Annika Bittmann, Aileen Mangang and Ramona Straub for overall excellent technical assistance. The neuroblastoma patient survival data were kindly provided by M. Fischer (University of Cologne, Germany). Support from the DKFZ Light Microscopy Facility is gratefully acknowledged. We would like to thank Nature Research Editing Service for editing and reviewing this manuscript for English language. This work was supported by the $\mathrm{H}$. W. \& J. Hector foundation \#M71 (IO) and Deutsche Forschungsgemeinschaft (DFG): MJ (Ju295/13-1), WS (Si868/13-1), OW (W1461/4-1) and IO (Oe542/2-1). JS received a DAAD scholarship for doctoral candidates. FRK was supported by the Deutsche Krebshilfe (DKH) with a Mildred Scheel doctoral scholarship (number 112065).

\section{Compliance with ethical standards}

Conflict of interest The authors declare that they have no conflict of interest.

Open Access This article is licensed under a Creative Commons Attribution 4.0 International License, which permits use, sharing, adaptation, distribution and reproduction in any medium or format, as long as you give appropriate credit to the original author(s) and the source, provide a link to the Creative Commons license, and indicate if changes were made. The images or other third party material in this article are included in the article's Creative Commons license, unless indicated otherwise in a credit line to the material. If material is not included in the article's Creative Commons license and your intended use is not permitted by statutory regulation or exceeds the permitted use, you will need to obtain permission directly from the copyright holder. To view a copy of this license, visit http://creativecommons. org/licenses/by/4.0/.

\section{References}

1. Matthay KK, Maris JM, Schleiermacher G, Nakagawara A, Mackall CL, Diller L, et al. Neuroblastoma. Nat Rev Dis Prim. 2016;2:16078.

2. Brodeur GM, Iyer R, Croucher JL, Zhuang T, Higashi M, Kolla V. Therapeutic targets for neuroblastomas. Expert Opin Ther Targets. 2014;18:277-92.

3. Kelly WK, O'Connor OA, Krug LM, Chiao JH, Heaney M, Curley $\mathrm{T}$, et al. Phase I study of an oral histone deacetylase inhibitor, suberoylanilide hydroxamic acid, in patients with advanced cancer. J Clin Oncol. 2005;23:3923-31.

4. de Ruijter AJ, van Gennip AH, Caron HN, Kemp S, van Kuilenburg $\mathrm{AB}$. Histone deacetylases (HDACs): characterization of the classical HDAC family. Biochem J. 2003;370:737-49.

5. Choudhary C, Kumar C, Gnad F, Nielsen ML, Rehman M, Walther TC, et al. Lysine acetylation targets protein complexes and co-regulates major cellular functions. Science. 2009;325: 834-40.

6. Glozak MA, Sengupta N, Zhang X, Seto E. Acetylation and deacetylation of non-histone proteins. Gene. 2005;363:15-23.

7. Buggy JJ, Sideris ML, Mak P, Lorimer DD, McIntosh B, Clark JM. Cloning and characterization of a novel human histone deacetylase, HDAC8. Biochem J. 2000;350:199-205. 
8. Somoza JR, Skene RJ, Katz BA, Mol C, Ho JD, Jennings AJ, et al. Structural snapshots of human HDAC8 provide insights into the class I histone deacetylases. Structure. 2004;12:1325-34.

9. Oehme I, Deubzer HE, Wegener D, Pickert D, Linke JP, Hero B, et al. Histone deacetylase 8 in neuroblastoma tumorigenesis. Clin Cancer Res. 2009;15:91-99.

10. Rettig I, Koeneke E, Trippel F, Mueller WC, Burhenne J, KoppSchneider A, et al. Selective inhibition of HDAC8 decreases neuroblastoma growth in vitro and in vivo and enhances retinoic acid-mediated differentiation. Cell Death Dis. 2015;6:e1657.

11. Iorns E, Lord CJ, Turner N, Ashworth A, Utilizing RNA. Interference to enhance cancer drug discovery. Nat Rev Drug Discov. 2007;6:556-68.

12. Walton JD, Kattan DR, Thomas SK, Spengler BA, Guo HF, Biedler JL, et al. Characteristics of stem cells from human neuroblastoma cell lines and in tumors. Neoplasia. 2004;6:838-45.

13. Krennhrubec K, Marshall BL, Hedglin M, Verdin E, Ulrich SM. Design and evaluation of 'Linkerless' hydroxamic acids as selective HDAC8 inhibitors. Bioorg Med Chem Lett. 2007;17: 2874-8.

14. Balasubramanian S, Ramos J, Luo W, Sirisawad M, Verner E, Buggy JJ. A novel histone deacetylase 8 (HDAC8)-specific inhibitor PCI-34051 induces apoptosis in T-cell lymphomas. Leukemia. 2008;22:1026-34.

15. Mi H, Muruganujan A, Casagrande JT, Thomas PD. Large-scale gene function analysis with the PANTHER classification system. Nat Protoc. 2013;8:1551-66.

16. Liu X, Lei M, Erikson RL. Normal cells, but not cancer cells, survive severe Plk1 depletion. Mol Cell Biol. 2006;26:2093-108.

17. Heald R, McLoughlin M, McKeon F. Human wee1 maintains mitotic timing by protecting the nucleus from cytoplasmically activated Cdc2 kinase. Cell. 1993;74:463-74.

18. Wang Y, Decker SJ, Sebolt-Leopold J. Knockdown of Chk1, Wee1 and Myt1 by RNA interference abrogates G2 checkpoint and induces apoptosis. Cancer Biol Ther. 2004;3:305-13.

19. Mosse YP, Laudenslager M, Longo L, Cole KA, Wood A, Attiyeh $\mathrm{EF}$, et al. Identification of ALK as a major familial neuroblastoma predisposition gene. Nature. 2008;455:930-5.

20. Heigener DF, Reck M. Crizotinib. Recent Results Cancer Res. 2014;201:197-205.

21. Butrynski JE, D'Adamo DR, Hornick JL, Dal Cin P, Antonescu CR, Jhanwar SC, et al. Crizotinib in ALK-rearranged inflammatory myofibroblastic tumor. N Engl J Med. 2010;363:1727-33.

22. Kwak EL, Bang YJ, Camidge DR, Shaw AT, Solomon B, Maki $\mathrm{RG}$, et al. Anaplastic lymphoma kinase inhibition in non-smallcell lung cancer. N Engl J Med. 2011;363:1693-703.

23. Kazandjian D, Blumenthal GM, Chen HY, He K, Patel M, Justice R, et al. FDA approval summary: crizotinib for the treatment of metastatic non-small cell lung cancer with anaplastic lymphoma kinase rearrangements. Oncologist. 2014;19:e5-11.

24. Mosse YP, Lim MS, Voss SD, Wilner K, Ruffner K, Laliberte J, et al. Safety and activity of crizotinib for paediatric patients with refractory solid tumours or anaplastic large-cell lymphoma: a Children's Oncology Group phase 1 consortium study. Lancet Oncol. 2013;14:472-80.

25. Schulte JH, Bachmann HS, Brockmeyer B, Depreter K, Oberthur A, Ackermann S, et al. High ALK receptor tyrosine kinase expression supersedes ALK mutation as a determining factor of an unfavorable phenotype in primary neuroblastoma. Clin Cancer Res. 2011;17:5082-92.

26. Molenaar JJ, Koster J, Ebus ME, van Sluis P, Westerhout EM, de Preter K, et al. Copy number defects of G1-cell cycle genes in neuroblastoma are frequent and correlate with high expression of E2F target genes and a poor prognosis. Genes Chromosomes Cancer. 2012;51:10-19.
27. Kocak H, Ackermann S, Hero B, Kahlert Y, Oberthuer A, Juraeva $\mathrm{D}$, et al. Hox-C9 activates the intrinsic pathway of apoptosis and is associated with spontaneous regression in neuroblastoma. Cell Death Dis. 2013;4:e586.

28. Wertman J, Veinotte CJ, Dellaire G, Berman JN. The Zebrafish xenograft platform: evolution of a novel cancer model and preclinical screening tool. Adv Exp Med Biol. 2016;916:289-314.

29. Heimburg T, Kolbinger FR, Zeyen P, Ghazy E, Herp D, Schmidtkunz K, et al. Structure-based design and biological characterization of selective histone deacetylase 8 (HDAC8) inhibitors with anti-neuroblastoma activity. J Med Chem. 2017;60:10188-204.

30. Hinson AR, Jones R, Crose LE, Belyea BC, Barr FG, Linardic CM. Human rhabdomyosarcoma cell lines for rhabdomyosarcoma research: utility and pitfalls. Front Oncol. 2013;3:183.

31. Berry T, Luther W, Bhatnagar N, Jamin Y, Poon E, Sanda T, et al. The ALK(F1174L) mutation potentiates the oncogenic activity of MYCN in neuroblastoma. Cancer Cell. 2012;22:117-30.

32. Tanaka N, Fukuzawa M. MYCN downregulates integrin alpha1 to promote invasion of human neuroblastoma cells. Int $\mathrm{J}$ Oncol. 2008;33:815-21.

33. Zhu S, Lee JS, Guo F, Shin J, Perez-Atayde AR, Kutok JL, et al. Activated ALK collaborates with MYCN in neuroblastoma pathogenesis. Cancer Cell. 2012;21:362-73.

34. Schulte JH, Lindner S, Bohrer A, Maurer J, De Preter K, Lefever S, et al. MYCN and ALKF1174L are sufficient to drive neuroblastoma development from neural crest progenitor cells. Oncogene. 2013;32:1059-65.

35. Muth D, Ghazaryan S, Eckerle I, Beckett E, Pohler C, Batzler J, et al. Transcriptional repression of SKP2 is impaired in MYCNamplified neuroblastoma. Cancer Res. 2010;70:3791-802.

36. Sattu K, Hochgrafe F, Wu J, Umapathy G, Schonherr C, Ruuth K, et al. Phosphoproteomic analysis of anaplastic lymphoma kinase (ALK) downstream signaling pathways identifies signal transducer and activator of transcription 3 as a functional target of activated ALK in neuroblastoma cells. FEBS J. 2013;280:5269-82.

37. Pei Y, Liu KW, Wang J, Garancher A, Tao R, Esparza LA, et al. HDAC and PI3K antagonists cooperate to inhibit growth of MYCdriven medulloblastoma. Cancer Cell. 2016;29:311-23.

38. Simmons JK, Michalowski AM, Gamache BJ, DuBois W, Patel J, Zhang $\mathrm{K}$, et al. Cooperative targets of combined mTOR/HDAC inhibition promote MYC degradation. Mol Cancer Ther. 2017;16:2008-21.

39. Bradner JE, West N, Grachan ML, Greenberg EF, Haggarty SJ, Warnow T, et al. Chemical phylogenetics of histone deacetylases. Nat Chem Biol. 2010;6:238-43.

40. Lambertz I, Kumps C, Claeys S, Lindner S, Beckers A, Janssens E, et al. Upregulation of MAPK negative feedback regulators and RET in mutant ALK neuroblastoma: implications for targeted treatment. Clin Cancer Res. 2015;21:3327-39.

41. Minucci S, Pelicci PG. Histone deacetylase inhibitors and the promise of epigenetic (and more) treatments for cancer. Nat Rev Cancer. 2006;6:38-51.

42. Yoo CB, Jones PA. Epigenetic therapy of cancer: past, present and future. Nat Rev Drug Discov. 2006;5:37-50.

43. Carpenter EL, Mosse YP. Targeting ALK in neuroblastoma-preclinical and clinical advancements. Nat Rev Clin Oncol. 2012;9:391-9.

44. Schonherr C, Ruuth K, Yamazaki Y, Eriksson T, Christensen J, Palmer RH, et al. Activating ALK mutations found in neuroblastoma are inhibited by crizotinib and NVP-TAE684. Biochem J. 2011;440:405-13.

45. Chen Y, Takita J, Choi YL, Kato M, Ohira M, Sanada M, et al. Oncogenic mutations of ALK kinase in neuroblastoma. Nature. 2008;455:971-4. 
46. Janoueix-Lerosey I, Lequin D, Brugieres L, Ribeiro A, de Pontual L, Combaret V, et al. Somatic and germline activating mutations of the ALK kinase receptor in neuroblastoma. Nature. 2008;455:967-70.

47. Bresler SC, Wood AC, Haglund EA, Courtright J, Belcastro LT, Plegaria JS, et al. Differential inhibitor sensitivity of anaplastic lymphoma kinase variants found in neuroblastoma. Sci Transl Med. 2011;3:108ra114.

48. George RE, Sanda T, Hanna M, Frohling S, Luther W 2nd, Zhang $\mathrm{J}$, et al. Activating mutations in ALK provide a therapeutic target in neuroblastoma. Nature. 2008;455:975-8.

49. De Brouwer S, De Preter K, Kumps C, Zabrocki P, Porcu M, Westerhout EM, et al. Meta-analysis of neuroblastomas reveals a skewed ALK mutation spectrum in tumors with MYCN amplification. Clin Cancer Res. 2010;16:4353-62.

50. Bresler SC, Weiser DA, Huwe PJ, Park JH, Krytska K, Ryles H, et al. ALK mutations confer differential oncogenic activation and sensitivity to ALK inhibition therapy in neuroblastoma. Cancer Cell. 2014;26:682-94.

51. Lamant L, Pulford K, Bischof D, Morris SW, Mason DY, Delsol $\mathrm{G}$, et al. Expression of the ALK tyrosine kinase gene in neuroblastoma. Am J Pathol. 2000;156:1711-21.

52. Passoni L, Longo L, Collini P, Coluccia AM, Bozzi F, Podda M, et al. Mutation-independent anaplastic lymphoma kinase overexpression in poor prognosis neuroblastoma patients. Cancer Res. 2009;69:7338-46.

53. Duijkers FA, Gaal J, Meijerink JP, Admiraal P, Pieters R, de Krijger RR, et al. Anaplastic lymphoma kinase (ALK) inhibitor response in neuroblastoma is highly correlated with ALK mutation status, ALK mRNA and protein levels. Cell Oncol. 2011;34:409-17.

54. Chakrabarti A, Oehme I, Witt O, Oliveira G, Sippl W, Romier C, et al. HDAC8: a multifaceted target for therapeutic interventions. Trends Pharmacol Sci. 2015;36:481-92.

55. Deardorff MA, Bando M, Nakato R, Watrin E, Itoh T, Minamino $\mathrm{M}$, et al. HDAC8 mutations in Cornelia de Lange syndrome affect the cohesin acetylation cycle. Nature. 2012;489:313-7.

56. Olson DE, Udeshi ND, Wolfson NA, Pitcairn CA, Sullivan ED, Jaffe JD, et al. An unbiased approach to identify endogenous substrates of "Histone" deacetylase 8. ACS Chem Biol. 2014;9:2210-6.

57. Wilson BJ, Tremblay AM, Deblois G, Sylvain-Drolet G, Giguere V. An acetylation switch modulates the transcriptional activity of estrogen-related receptor alpha. Mol Endocrinol. 2010;24:1349-58.

58. Scholz C, Weinert BT, Wagner SA, Beli P, Miyake Y, Qi J, et al. Acetylation site specificities of lysine deacetylase inhibitors in human cells. Nat Biotechnol. 2015;33:415-23.

59. Zhang L, Yue Y, Ouyang M, Liu H, Li Z. The Effects of IGF-1 on TNF-alpha-treated DRG neurons by modulating ATF3 and GAP43 expression via PI3K/Akt/S6K signaling pathway. Neurochem Res. 2017;42:1403-21.
60. Love JM, Bober BG, Orozco E, White AT, Bremner SN, Lovering $\mathrm{RM}$, et al. mTOR regulates peripheral nerve response to tensile strain. J Neurophysiol. 2017;117:2075-84.

61. Mutoh T, Rudkin BB, Koizumi S, Guroff G. Nerve growth factor, a differentiating agent, and epidermal growth factor, a mitogen, increase the activities of different S6 kinases in PC12 cells. J Biol Chem. 1988;263:15853-6.

62. Armijo-Weingart L, Gallo G. It takes a village to raise a branch: cellular mechanisms of the initiation of axon collateral branches. Mol Cell Neurosci. 2017;84:36-47.

63. Nieto-Estevez V, Defterali C, Vicario-Abejon C. IGF-I: a key growth factor that regulates neurogenesis and synaptogenesis from embryonic to adult stages of the brain. Front Neurosci. 2016;10:52.

64. Zhang X, He X, Li Q, Kong X, Ou Z, Zhang L, et al. PI3K/AKT/ mTOR signaling mediates valproic acid-induced neuronal differentiation of neural stem cells through epigenetic modifications. Stem Cell Rep. 2017;8:1256-69.

65. Oehme I, Linke JP, Bock BC, Milde T, Lodrini M, Hartenstein B, et al. Histone deacetylase 10 promotes autophagy-mediated cell survival. Proc Natl Acad Sci USA. 2013;110:E2592-2601.

66. Dreidax D, Bannert S, Henrich KO, Schroder C, Bender S, Oakes $\mathrm{CC}$, et al. p19-INK4d inhibits neuroblastoma cell growth, induces differentiation and is hypermethylated and downregulated in MYCN-amplified neuroblastomas. Hum Mol Genet. 2014;23:6826-37.

67. De Brouwer S, De Preter K, Kumps C, Zabrocki P, Porcu M, Westerhout EM, et al. Meta-analysis of neuroblastomas reveals a skewed ALK mutation spectrum in tumors with MYCN amplification. Clin Cancer Res. 2010;16:4353-62.

68. Pelz O, Gilsdorf M, Boutros M. Web cell HTS2: a webapplication for the analysis of high-throughput screening data. BMC Bioinform. 2010;11:185.

69. Oehme I, Bosser S, Zornig M. Agonists of an ecdysone-inducible mammalian expression system inhibit Fas ligand- and TRAILinduced apoptosis in the human colon carcinoma cell line RKO. Cell Death Differ. 2006;13:189-201.

70. Fischer M, Skowron M, Berthold F. Reliable transcript quantification by real-time reverse transcriptase-polymerase chain reaction in primary neuroblastoma using normalization to averaged expression levels of the control genes HPRT1 and SDHA. J Mol Diagn. 2005;7:89-96.

71. Ritchie ME, Phipson B, Wu D, Hu Y, Law CW, Shi W, et al. Llimma powers differential expression analyses for RNAsequencing and microarray studies. Nucleic Acids Res. 2015; 43:e47

72. Fior R, Povoa V, Mendes RV, Carvalho T, Gomes A, Figueiredo $\mathrm{N}$, et al. Single-cell functional and chemosensitive profiling of combinatorial colorectal therapy in zebrafish xenografts. Proc Natl Acad Sci USA. 2017;114:E8234-E8243. 